Check for updates

Cite this: J. Mater. Chem. C, 2021 , 9, 10573

Received 5th April 2021,

Accepted 25th May 2021

DOI: 10.1039/d1tc01576a

rsc.li/materials-c

\section{Spin-ladder behaviour in molecular materials $\dagger$}

\begin{abstract}
Rafaela A. L. Silva (iD ${ }^{a}$ and Manuel Almeida (D) *ab
Spin-ladders are quantum magnetic systems at the crossroads between one and two dimensions that have attracted increasing interest during the last decades due to unique properties critically dependent on spin system topology. The first materials where spin-ladder behaviour was identified were transition metal oxides and copper(II) halides or coordination polymers. However, since the report of the first spinladder based in an organic molecule in 1997, an increasing number of molecular compounds showing spin-ladder behaviour have been reported. Their structural and magnetic properties are reviewed with emphasis on the possibilities offered by molecular units of different nature and crystal engineering tools to develop new spin-ladder systems.
\end{abstract}

\section{Introduction}

Spin-ladders are a class of low dimensional magnetic systems that consist of a finite number of magnetically coupled spins chains. These systems can be seen as intermediate between the strict 1D (one dimensional) model of an isolated chain and a layer in a 2D (two dimensional) magnetic lattice (Fig. 1) and recently have attracted an increasing attention under the more general context of low dimensional and topological quantum systems. ${ }^{1-3}$ They are particularly interesting as a result of the

${ }^{a}$ C2TN, Instituto Superior Técnico, Universidade de Lisboa, CTN, EN 10, P-2695-066 Bobadela LRS, Portugal. E-mail: malmeida@ctn.tecnico.ulisboa.pt

${ }^{b}$ Departament of Nuclear Sciences and Engineering, Instituto Superior Técnico, Universidade de Lisboa, E.N. 10, P-2695-066 Bobadela LRS, Portugal

$\dagger$ This paper is part of a Journal of Materials Chemistry C themed issue in honour of Professor Jaume Veciana and Professor Concepció Rovira. increased importance of the quantum mechanical effects operating within them and their properties were found critically dependent on the specific topology, symmetry and spin values. These effects make the evolution of the magnetic properties from 1D chains towards a 2D plane, not at all continuous as one could naively predict.

Theoretical predictions and experimental verifications show that depending on the number of interacting spin chains (legs in the spin-ladder) quite different magnetic behaviour may be observed. ${ }^{1-4}$ Antiferromagnetic (AF) coupled $S=\frac{1}{2}$ spin-ladders with an odd number of legs behave as an isolated chain with a finite susceptibility upon approaching zero temperature and a power-law fall off of the spin-correlations. This contrasts with spin-ladders with an even number of legs that present a gap in the magnetic excitations, making the susceptibility to drop exponentially towards zero upon cooling, in a spin-liquid ground

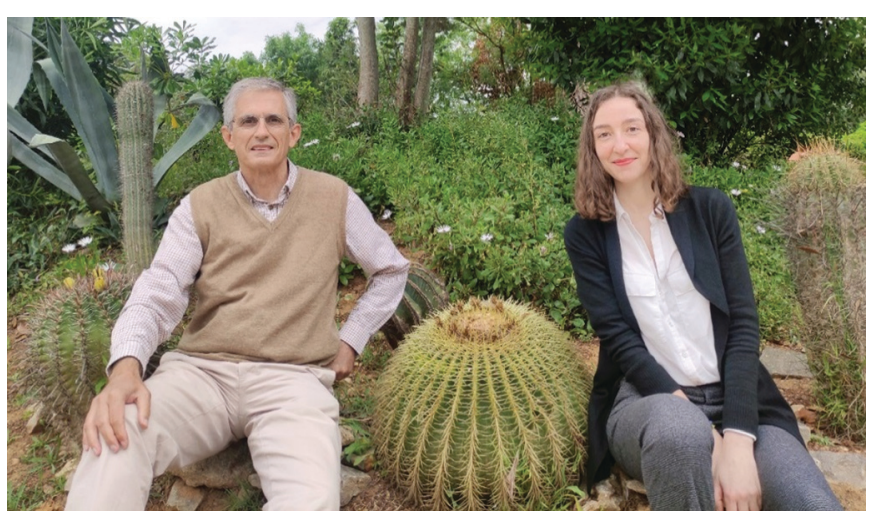

Manuel Almeida (left) and Rafaela A. L. Silva (right)
Rafaela Silva obtained her PhD in Chemistry in 2017 from Instituto Superior Técnico (IST), Universidade de Lisboa, under supervision of Prof. Manuel Almeida. She currently is a research assistant in the Solid State Group@C2TN-IST focusing her research interests in molecular design and synthesis of molecular materials with unconventional electric properties.

Prof. Manuel Almeida studied at the Technical University of Lisbon where he obtained a PhD in 1984 under supervision of Prof. Luis Alcácer. After a postdoctoral stay with Prof. Tobin Marks at the Northwestern University, USA, during 1985-1986, he joined the Portuguese National Laboratory LNETI to establish a research group on Solid State Physics and Chemistry, which was focused on preparation and characterization of materials with unconventional electrical and magnetic properties. His laboratory was since 2013 integrated in the University of Lisbon where he is leading research activities in materials chemistry and physics. 


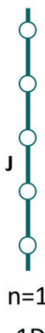

1D

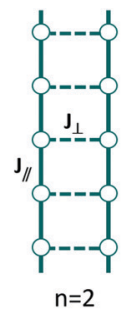

$n=2$

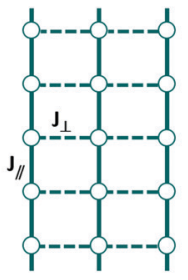

$n=3$

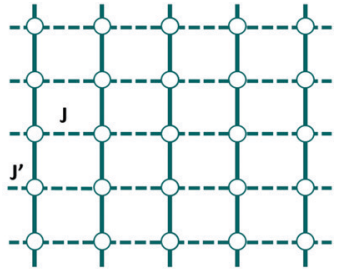

2D
Fig. 1 Schematic representation of low dimensional magnetic Heisenberg systems with exchange coupling constants $J$, specifically of a 1D chain $(n=1)$, two- $(n=2)$ and three-legged $(n=3)$ spin-ladder systems and 2D square lattice. White circles represent $S=\frac{1}{2}$ spins and continuous and dashed lines denote AF interactions of different strength.

state lacking long-range order. The even-leg ladders can be seen as spin singlet pairs with a spin-spin correlation length along the legs showing an exponential decay produced by the presence of a finite spin gap. The situation is also quite different depending on the spin values and for instance, as first pointed out by Haldane, ${ }^{5}$ a chain of integer spins presents a gap in the magnetic excitations, as the $S=\frac{1}{2}$ spin-ladder with even legs, at variance with the $S=\frac{1}{2} \mathrm{AF}$ Heisenberg chain.

Besides the fundamental physics aspects related with these fascinating quantum and topological effects, the interest in spin-ladders has been also motivated by the theoretical prediction that upon light hole doping they can become superconducting due spin mediated hole-hole attraction. ${ }^{1,6}$ This possibility, similar to the cuprate oxides where the high-temperature superconductivity arrives near an AF state, may open a new strategy for the development of new superconducting materials.

\section{Main features of spin-ladders}

The two leg ladder with isotropic AF coupling constants (Heisenberg AF spin-ladders), can be described by the following Hamiltonian:

$$
H=J_{\|} \sum_{a=1,2} \sum_{i=1}^{L} S_{i, a} \cdot S_{i+1, a}+J_{\perp} \sum_{i=1}^{L} S_{i, 1} \cdot S_{i, 2}
$$

where $S_{i, a}$ are the spin operators at site $i(i=1,2, \ldots L)$ of leg $a$ $(1,2)$ of a ladder with $L$ rungs. $J_{\|}$and $J_{\perp}$ are the intra- and interrung exchange couplings respectively, which are positive for $\mathrm{AF}$ interactions. $^{1}$

The magnetic properties of the spin-ladders have been discussed considering situations of experimental interest with intra- and interchain couplings of the same order of magnitude $\left(J_{\|} \approx J_{\perp}\right)$, that is intermediate between the extreme cases of $J_{\perp} / J_{\|}=0$ and $J_{\|} / J_{\perp}=0$, corresponding to isolated $1 \mathrm{D}$ chains and isolated dimers, respectively, which are well understood. While the situations close to these extreme cases, can be treated by perturbative calculations, for the more interesting real situations $\left(J_{\|} \approx J_{\perp}\right)$ there are no known exact solutions. These situations have been discussed by exact diagonalization of small clusters or by numerical simulations using Quantum Monte Carlo (QMC) techniques. ${ }^{7-9}$ It should be kept in mind

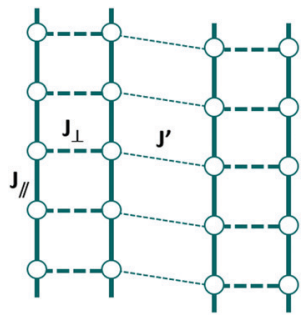

Fig. 2 Schematic representation of interladder couplings $J^{\prime}$.

that in real materials the ladders are never entirely isolated and often additional interladder couplings $J^{\prime}$ have to be taken into account (Fig. 2). These interladder interactions, if significant, can easily destroy the spin-liquid state promoting a 3D (three dimensional) AF ordered state. The critical value of the ratio of inter- to intra-ladder interactions that is capable to induce a $3 \mathrm{D}$ ordered sate is estimated as of the order of $J^{\prime} / J_{\perp} \approx 0.1 .^{10}$

The characterisation of the spin-ladder systems in a first stage has been done mainly by analysis of the temperature and field dependence of the magnetic susceptibility, enabling to extract fundamental parameters such as effective exchange couplings, $J$, and spin excitation gap, $\Delta$.

For a two leg $S=1 / 2$ AF ladder at low temperatures, below the excitation gap, $\Delta$, the magnetic susceptibility, $\chi_{\text {ladder }}$, follows an exponential decay given by the Troyer expression ${ }^{11}$

$$
\chi_{\text {ladder }}=\alpha T^{-1 / 2} \exp \left(-\Delta / k_{\mathrm{B}} T\right)
$$

where $k_{\mathrm{B}}$ is the Boltzmann constant, $\alpha$ is a constant corresponding to the dispersion of the excitation energy, and $\Delta$ is the energy gap in the spin-excitation spectrum.

In a more extended temperature range, $\chi_{\text {ladder }}$ presents a broad maximum at temperatures above the gap, following a behaviour that can be approximated by the parameterization eqn (3) given by Barnes and Riera, as a function of $J_{\|}$and $J_{\perp}:{ }^{10}$

$$
\chi_{\text {ladder }}=\frac{c_{1}}{T}\left[1+\left(\frac{T}{c_{2}}\right)^{c_{3}}\left(\mathrm{e}^{\frac{c_{4}}{T}}-1\right)\right]^{-1}\left[1+\left(\frac{c_{5}}{T}\right)^{c_{6}}\right]^{-1}
$$

where,

$$
c_{1}=N g^{2} \mu_{\mathrm{B}}^{2} / 4 k_{\mathrm{B}}
$$

$N$ is the Avogadro's number, $g$ the Lande $g$-factor, $\mu_{\mathrm{B}}$ the Bohr magneton and parameters $C_{2}, C_{3}, C_{4}, C_{5}$ and $C_{6}$, are function of the exchange constants $J_{\perp}$ and $J_{\|}$. The spin gap $\Delta$ can also be estimated from the resulting values of $J_{\|}$and $J_{\perp}$ with the theoretical equation ${ }^{10,11}$

$$
\Delta=J_{\perp}-J_{\|}+J_{\|}^{2} / 2 J_{\perp}
$$

Considering the situation $J_{\perp}>J_{\|}$of the theoretical model of Troyer et al., ${ }^{11}$ the coupling parameters are related with the temperature of maximum susceptibility by the following quadratic expression:

$$
\frac{T\left(\chi_{\mathrm{m}^{\prime} \mathrm{ax}}\right)}{J_{\perp}}=0.625-0.01835 \frac{J_{\|}}{J_{\perp}}+0.2532\left(\frac{J_{\|}}{J_{\perp}}\right)^{2}
$$

The magnetic exchange coupling constants $J$ between two radicals can be calculated with fair approximation using sophisticated 
first-principles quantum chemistry calculations as for instance based on unrestricted density functional theory (DFT). However, for comparison purposes reliable conclusions and semi quantitative estimates can be obtained using simpler calculations methods. According to the Hubbard model the AF exchange coupling constant $J$ between two radicals can be related to the ratio:

$$
J=2 t^{2} / U
$$

where $t$ is the transfer integral between the single occupied molecular orbitals (SOMO) and $U$ the on-site electron Coulomb repulsion energy. ${ }^{12}$ The absolute values of $J$ cannot be accurately obtained using this equation because $U$, typically of the order of $1 \mathrm{eV}$ for organic radicals, cannot be easily obtained in a reliable manner. However, this equation is very useful to analyse the relative magnitude of the different interactions $J$ from the transfer integrals $t$ that for comparison purposes can be calculated even using simple tight-binding methods.

While the exchange coupling parameters can be obtained from analysis of the experimental magnetisation data or from theoretical calculations, they are not enough to characterise a compound as a spin-ladder system. A possible 3D magnetic ordering at low temperature due to interladder coupling interactions $J^{\prime}$ and other sources for the opening of a gap in the spin excitations must be excluded. Therefore, additional experiments with microscopic probes such as muon spin resonance $\left(\mu^{+} \mathrm{SR}\right)$, nuclear magnetic resonance (NMR) or neutron scattering, are required for determination of the exact nature of the ground state as a spin-ladder without long range order.

Under magnetic field $\mathbf{h}$ an additional Zeeman energy term in the Hamiltonian of eqn (1), $-g \mu_{\mathrm{B}} \mathbf{h} \cdot S^{z}{ }_{i}$, $g$ is the $g$-factor and $\mu_{\mathrm{B}}$ the Bohr magneton), must be considered for each spin $i$. The main effect of a large magnetic field is to reduce the spin gap until a quantum critical point, at $h_{\mathrm{c} 1}$, separating the low field disordered gaped state from a gap-less state, which in ladders is a spin Luttinger-liquid with algebraically decaying spin correlations. At a higher field, $h_{\mathrm{c} 2}$, a second quantum critical transition is expected to occur to a state with full polarisation of the spins (magnetisation saturation). ${ }^{13}$ In the partially polarized region between $h_{\mathrm{c} 1}$ and $h_{\mathrm{c} 2}$ it has been suggested that weak interladder coupling may easily induce at lower temperatures a 3D condensation of magnetic excitations. ${ }^{14-16}$ These effects are currently under debate and their experimental observation is dependent on the availability of magnetic fields large enough when compared with the spin gaps and in practical terms has been so far restricted to only a few compounds. ${ }^{13,17}$

\section{Realisation of spin-ladders}

The spin-ladder behaviour was first described in transition metal oxides $(\mathrm{Cu} \text { and } \mathrm{V})^{10,18,19}$ and copper(II) halides, ${ }^{20-23}$ although some of them like $\mathrm{LaCuO}_{2.5}{ }^{24,25}$ and $(\mathrm{VO}){ }_{2} \mathrm{P}_{2} \mathrm{O}_{7}{ }^{26}$ were latter not confirmed as real spin-ladders as they undergo ordering transitions at low temperatures.

Spin-ladder behaviour based on an organic species was first identified in (DT-TTF $)_{2}\left[\mathrm{Au}(\mathrm{mnt})_{2}\right](\mathbf{1}),{ }^{27}$ and its report stimulated the search for other molecule based spin ladder-systems. In the context of the development of new spin-ladders, the wide range of possible chemical modifications and structural flexibility offered by molecular chemistry, together with crystal engineering principles, make the molecular materials very appealing, rising the hopes for achieving a rational design of new or improved magnetic systems. The strategy for building spin-ladders from spin carrying molecular units can be conceptually envisaged either by assembling magnetic chains in pairs or by assembling chains of paramagnetic dimers as previously pointed out in early reviews of this topic. ${ }^{28-30}$ In spite of these general guiding rules, the molecular compounds showing spin-ladder behaviour have been obtained largely by serendipity. They include a large diversity of molecular spin carrying units, from electroactive molecules derived from TTF or transition metal bisdisdithiolene complexes, to other organic radicals with light hetero atoms such as nitroxyls, verdazyls and bisdithiazolyls. In a few cases the compounds are structurally related.

Table 1 lists different molecular compounds that have been reported with a spin-ladder behaviour and their experimentally derived magnetic parameters.

The present review is focused on spin-ladder systems of organic or molecular nature, where the magnetic moments are associated with molecular orbitals, excluding other inorganic systems where the magnetic moments are essentially localised in metal centres such as oxides or coordination polymers, including a large number of $\mathrm{Cu}$ (II) halides that have been subject of a recent specialised review. ${ }^{31}$

The discussion of the properties of these compounds will be organised according to the type of spin carrying units, with special emphasis to the compounds that are structurally closely related, where the effects of chemical and structural modifications in their properties can more easily be placed into evidence.

\section{1. (DT-TTF $)_{2}\left[\mathrm{Au}(\mathrm{mnt})_{2}\right]$; the first organic spin-ladder}

$(\mathrm{DT}-\mathrm{TTF})_{2}\left[\mathrm{Au}(\mathrm{mnt})_{2}\right]$ (1) (Scheme 1) was the first molecular material reported with a spin-ladder behaviour entirely due to the organic units, DT-TTF (dithiophene tetrathiafulvalene), a derivative of the organic donor TTF (tetrathiafulvalene). ${ }^{27}$ This compound was the subject of detailed studies using different techniques that are summarised in this section, confirming it as a spin-ladder system. The discovery of spin-ladder behaviour in 1 stimulated the search and discovery of several closely related compounds also with spin-ladder behaviour which are described in the next section.

The crystal structure of $\mathbf{1}$ consists in chains of donor molecules and chains of diamagnetic anions both regularly stacked along the $b$ axis (Fig. 3). ${ }^{27}$ The donor and anion chains alternate in the $(a, c)$ plane in such a way that donor chains are arranged in tightly connected pairs, surrounded by chains of anions and only loosely connected to other pairs of donor chains.

The two donor chains in a pair are related by a screw axis and connected by several short $\mathrm{S} \cdot \mathrm{S}$. contacts, below the sum of the van der Waals radii (Fig. 3b). While the anions are diamagnetic closed shell species, the stoichiometry imposes 
Table 1 Experimental spin-ladder parameters in different compounds

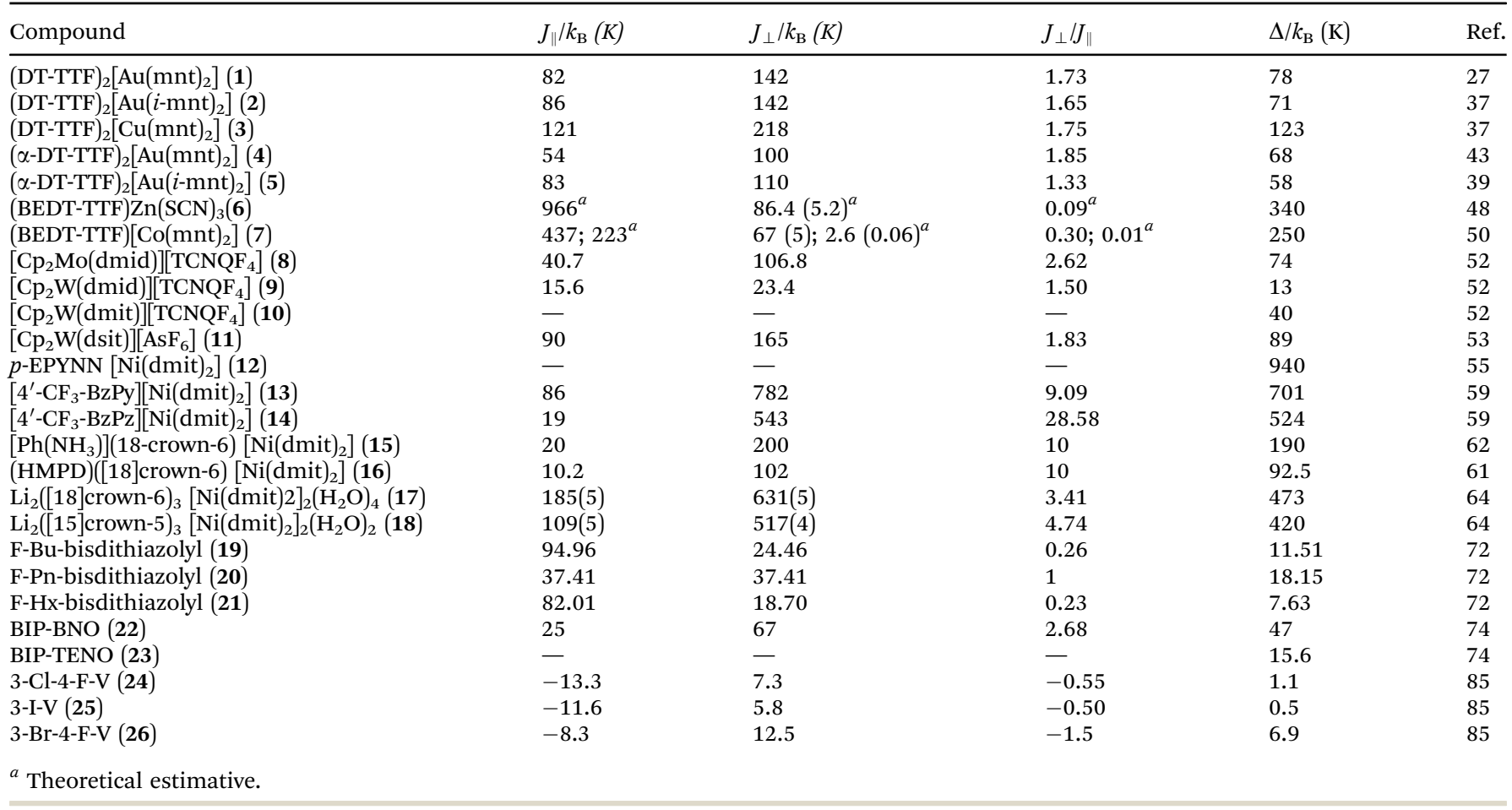

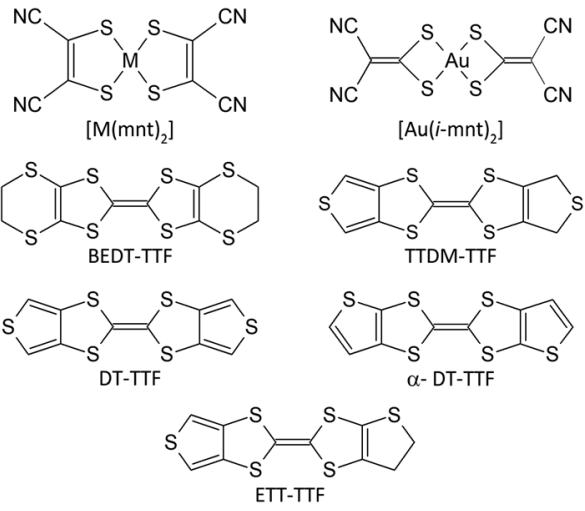

Scheme 1 Molecular building blocks of TTF based spin-ladders.

that the donors are partially oxidised, in average as (DT-TTF) ${ }^{0.5+}$, the paramagnetic properties of this compound (Fig. 4) being therefore entirely due to the unpaired electrons in the organic donor chains.

In the structure of $\mathbf{1}$ there are 3 types of interdonor contacts associated with distinct highest occupied molecular orbital (HOMO) intermolecular interactions; the largest one estimated as $t_{1} \sim 36 \mathrm{meV}$ along the stacks, due to the $\pi-\pi$ overlap of donor orbitals; $t_{2} \sim 21 \mathrm{meV}$ between molecules in paired chains associated with short S $\cdots \mathrm{S}$ contacts and the smallest one $t_{3} \sim$ $6 \mathrm{meV}$ which, through the terminal $\mathrm{S}$ atom, connects molecules in two different pairs of chains. ${ }^{27}$ The modest magnitude of $t_{1}$ when compared with typical molecular conductors with metallic properties, suggests the partially oxidized unpaired electrons in donor chains are localized due to significant electron repulsion

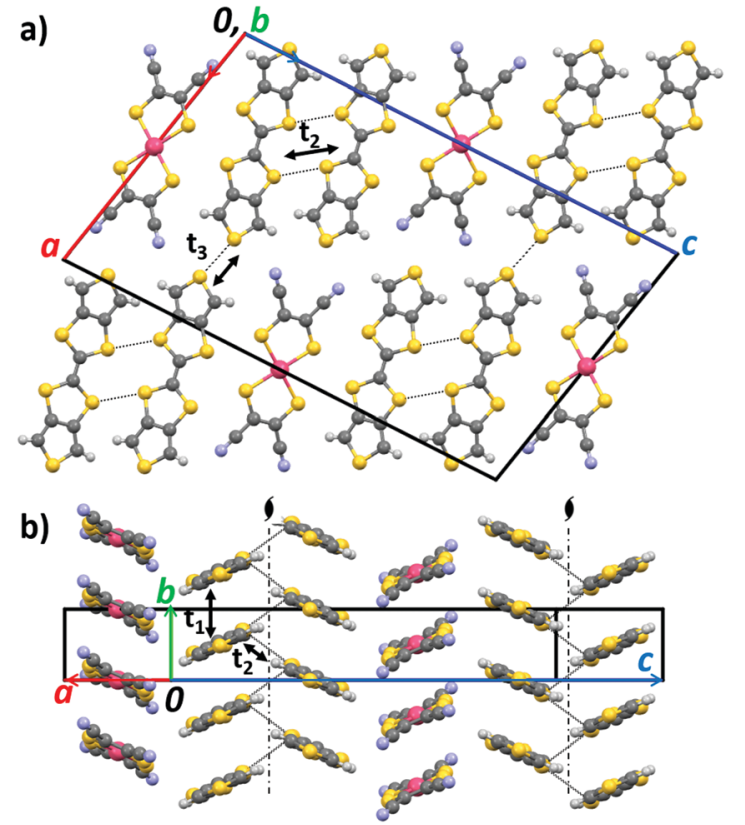

Fig. 3 Crystal structure of (DT-TTF) ${ }_{2}\left[\mathrm{Au}(\mathrm{mnt})_{2}\right]$ (1): (a) view along the stacking axis $b$; (b) partial view of layers of neighbouring donor and acceptor chains along their molecular chain axis.

interactions, in agreement with the thermally activated semiconducting regime of the electrical conductivity with $8 \mathrm{~S} / \mathrm{cm}$ at room temperature (Fig. 4). ${ }^{27,32}$ This localized regime of electrons in the donor chains is also confirmed by pressure studies of the electrical resistivity showing that a metallic 


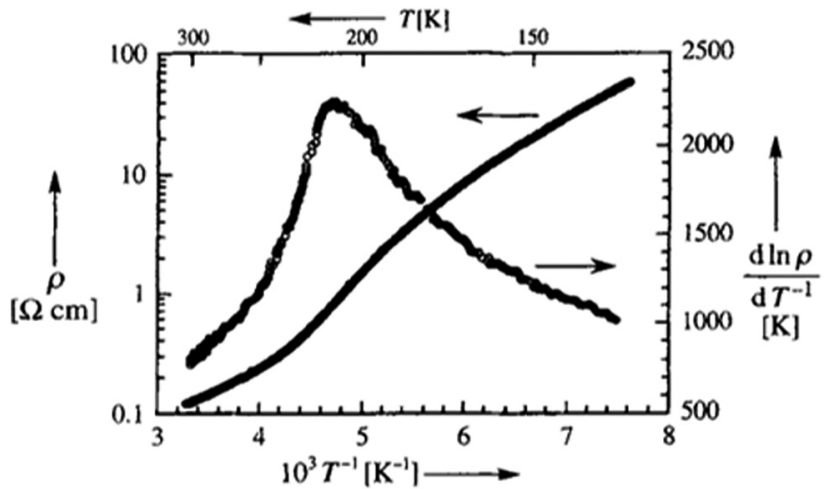

Fig. 4 Electrical resistivity $\rho$ of (DT-TTF) $)_{2}\left[\mathrm{Au}(\mathrm{mnt})_{2}\right](\mathbf{1})$ as a function of the reciprocal temperature (left) and its first derivative, $\mathrm{d} \ln \rho / \mathrm{d} T^{-1}$ (right). Reproduced with permission from ref. 27. Copyright 1997 Wiley-VCH.

regime is only achieved at pressures above $\approx 15 \mathrm{Kbar}$, when increased interactions and associated bandwidth can overcome the electronic repulsion energy. ${ }^{33}$ The activation energy of the conductivity at ambient pressure is not constant and presents a broad maximum around $220 \mathrm{~K}$ (Fig. 4). X-ray diffraction experiments have shown that this anomaly at $\approx 220 \mathrm{~K}$ is associated with the enhancing and sharpening of diffuse scattering planes centered at $0.5 b^{*}$, ascribed to a dimerization of the donor chains, at a local scale and without long range order (Fig. 5). The average domains size of the chain dimerization, estimated from the width of the diffuse scattering planes, increases upon cooling from room temperature, saturating below $180 \mathrm{~K}$ at about $150 \AA$ (i.e. $40 b$ ) as shown in (Fig. 6). ${ }^{27}$

Therefore, dimer units (DT-TTF $)_{2}{ }^{\bullet+}$ were identified as spin carrying units in this ladder structure of $\mathbf{1}$. Although initially not clear the detailed microscopic mechanism of this dimerisation, infrared spectroscopy studies show a splitting of several donor vibration modes below $220 \mathrm{~K},{ }^{34}$ suggesting that the dimerization could be associated with donor charge disproportionation. This was later confirmed to be the case in the $\mathrm{Cu}$ analogue by the determination of the crystal structure at low temperature (vide next section).

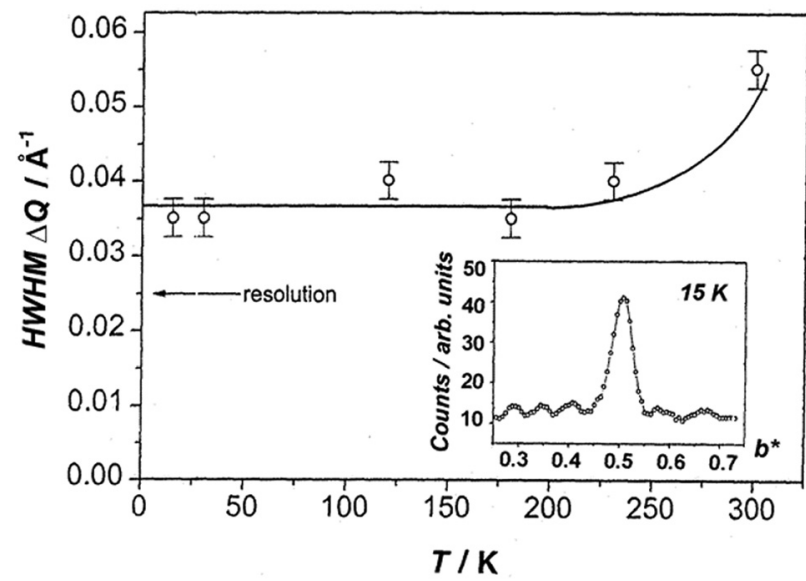

Fig. 5 Thermal dependence of the HWHM along the $b$ axis of the $1 / 2 b^{*}$ diffuse lines of 1 . The inset shows the profile along $b^{*}$ of such a line. Reproduced with permission from ref. 27. Copyright 1997 Wiley-VCH.

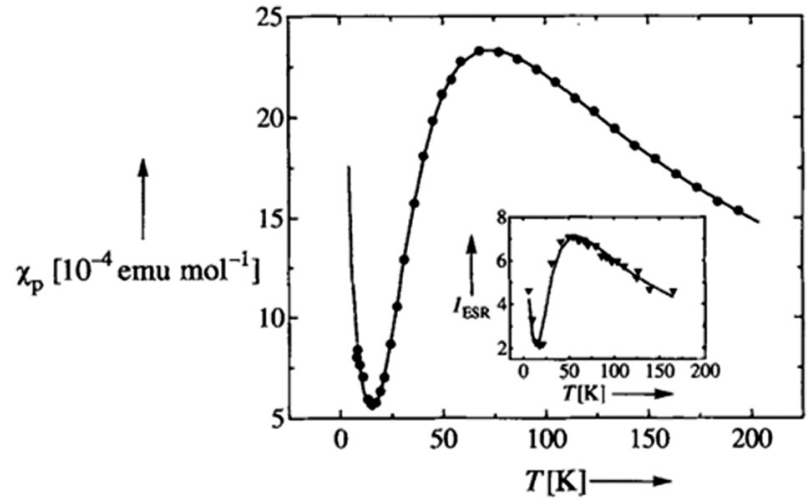

Fig. 6 Temperature dependence of the paramagnetic susceptibility of (DT-TTF) ${ }_{2}\left[\mathrm{Au}(\mathrm{mnt})_{2}\right](\mathbf{1})$. The solid line is the fit with eqn (3). Inset shows the temperature dependence of paramagnetic susceptibility measured by ESR on a single crystal. Reproduced with permission from ref. 27. Copyright 1997 Wiley-VCH.

The ESR spectrum consists in a single line with average $g$ value close to that of the cation radical $[\mathrm{DT}-\mathrm{TTF}]^{+}$in solution and the spin susceptibility obtained by integration of EPR signal, follows the same temperature dependence as the static paramagnetic susceptibility (Fig. 6) confirming that the paramagnetic properties of $\mathbf{1}$ are entirely due to the organic donors. Besides a Curie tail corresponding to about $2 \%$ of $S=\frac{1}{2}$ impurities or defects, the paramagnetic susceptibility presents a typical spin-ladder behaviour with a maximum at about $70 \mathrm{~K}$ and an exponential decrease towards zero upon cooling at lower temperatures. The susceptibility data in the range 8 to $45 \mathrm{~K}$ could be fitted by eqn (2), with a gap $\Delta=78 \mathrm{~K}$, and the data up to room temperature was fitted by eqn (3) with $J_{\perp} / k_{\mathrm{B}}=142 \mathrm{~K}$ and $J_{\|} / k_{\mathrm{B}}=83 \mathrm{~K}^{27}$

Zero-field and longitudinal field $\mu^{+} \mathrm{SR}$ measurements as a function of temperature were performed confirming the absence of magnetic order down to $85 \mathrm{mK}$, thus supporting its description of $\mathbf{1}$ as a two-leg spin-ladder with a quantum spin-liquid ground state, despite the small ladder-ladder interactions. ${ }^{35}$

\subsection{Spin-ladders derived from (DT-TTF $)_{2}\left[\mathrm{Au}(\mathrm{mnt})_{2}\right]$}

Soon after the report of $\mathbf{1}$ as the first organic spin-ladder compound, a comprehensive exploration of related compounds, based on modifications of either the donor or anions was undertaken. ${ }^{32,36,37}$ As a result, several closely related compounds with donor ladder structure were obtained, thus originating a relatively large family of isostructural or closely related spinladder compounds. The comparison of the compounds in this family provides useful insights on how the spin-ladder properties are controlled by different key molecular and crystalline structural effects.

In a first strategy to obtain new spin-ladder compounds a large number of diamagnetic monoanionic transition metal (Au and $\mathrm{Cu}$ ) bisdithiolene complexes, with similar ligands, were considered as substitutes for $\left[\mathrm{Au}(\mathrm{mnt})_{2}\right]$. However, it was found that unless the complexes were very closely related and with comparable size, completely different structures were obtained. 
Compounds isostructural to $\mathbf{1}$, with donor arranged in paired stacks, could be obtained only with the quite similar anions $\left[\mathrm{Cu}(\mathrm{mnt})_{2}\right]^{-}$and $\left[\mathrm{Au}(i \text {-mnt })_{2}\right]^{-}$. These anions are known to be not very stable in solution and both (DT-TTF $)_{2}\left[\mathrm{Au}(i \text {-mnt })_{2}\right]$ (2) and $(\mathrm{DT}-\mathrm{TTF})_{2}\left[\mathrm{Cu}(\mathrm{mnt})_{2}\right]$ (3) were obtained as single crystals by electrocrystallisation from the corresponding tetrabutylammonium anion salts, only in small amounts. ${ }^{37}$

$(\mathrm{DT}-\mathrm{TTF})_{2}\left[\mathrm{Au}(i \text {-mnt })_{2}\right]$ (2) was obtained as a minority phase among two other compounds with a different $\mathrm{Au}(\mathrm{I})$ dimeric core anion generated in situ, (DT-TTF $)_{9}\left[\mathrm{Au}_{2}(i \text {-mnt })_{2}\right]_{2}$ and (DT-TTF $)_{2}$ $\left[\mathrm{Au}_{2}(i \text {-mnt })_{2}\right]$. (DT-TTF $)_{2}\left[\mathrm{Cu}(\mathrm{mnt})_{2}\right](3)$ was often obtained together with fully ionic salt (DT-TTF) $\left[\mathrm{Cu}(\mathrm{mnt})_{2}\right] \cdot{ }^{37}$ Due to these difficulties in obtaining single phase material in large amount, magnetic susceptibility of 2 could be determined only by integration of ESR signal. Its temperature dependence follows a spin ladder behaviour identical to that of the $\left[\mathrm{Au}(\mathrm{mnt})_{2}\right]$ analogue, with the parameters $\Delta / k_{\mathrm{B}}=71 \mathrm{~K}, J_{\|} / k_{\mathrm{B}}=86 \mathrm{~K}$ and $J_{\perp} / k_{\mathrm{B}}=142 \mathrm{~K}$ almost identical to those of (DT-TTF $)_{2}\left[\mathrm{Au}(\mathrm{mnt})_{2}\right]$, which is not surprising in view of the very similar crystal structure with virtually identical interdonor distances. ${ }^{37}$

Reliable magnetic susceptibility data for $\mathbf{3}$, could be obtained only by EPR and found to follow a similar spin-ladder behaviour, but with slightly larger parameters $\Delta / k_{\mathrm{B}}=123 \mathrm{~K}, J_{\|} / k_{\mathrm{B}}=121 \mathrm{~K}$ and $J_{\perp} / k_{\mathrm{B}}=218 \mathrm{~K}$. The larger magnetic exchange coupling parameters in the $\mathrm{Cu}$ compound result from a tighter packing of the donors as consequence of the smaller size of the anion.

An important characteristic of the $\mathrm{Cu}$ compound 3 compared with the $\mathrm{Au}$ analogue $\mathbf{1}$ is a sharper transition at $235 \mathrm{~K}$, seen in electrical resistivity as a sharp maximum in $\mathrm{d} \ln \rho / \mathrm{d} T^{-1}$. This transition was found to be associated with the appearance of weak but sharp X-ray diffraction spots corresponding to a lattice doubling along the chain axis $b$ with 3D long range order. This situation contrasts with the diffuse lines observed in the $\mathrm{Au}$ analogue bellow a broader transition centered at $220 \mathrm{~K}$. The superlattice spots in the $\mathrm{Cu}$ compound are sharp but rather weak, they were first observed in a sensitive experimental set up to study diffuse scattering ${ }^{38}$ but could not be detected with standard laboratory diffractometers even using long measuring time. However, using synchrotron radiation at ESRF they are clearly visible in very small crystals below $235 \mathrm{~K}$ allowing to collect a full diffraction data set and to solve the superstructure at $120 \mathrm{~K}^{39}$ This low temperature superstructure is triclinic, space group $P \overline{1}$, with doubling of the lattice parameter $b$ along the stacks and loss of the screw axis relating the paired donor chains. This doubling is due to a charge ordering scheme with alternating neutral and charged molecules, as denoted by the donor bond lengths, along the chain axis $b$ (Fig. 7c). This ordering scheme is a realization of the Bond-Charge-Density Wave broken symmetry state previously suggested for $\frac{1}{4}$ filled zig-zag ladders ${ }^{40}$ and in agreement with previous infra-red spectroscopy studies suggesting charge ordering in this compound $^{41}$ as well as in the Au analogue. ${ }^{34}$ It entirely rules out the possible alternative dimerisation arrangement based on a bond ordering scheme (Fig. 7b). The charge ordering scheme also explains why the ladders in this family of compounds are of strong rung type; $J_{\perp}$ is an interaction between spins localised
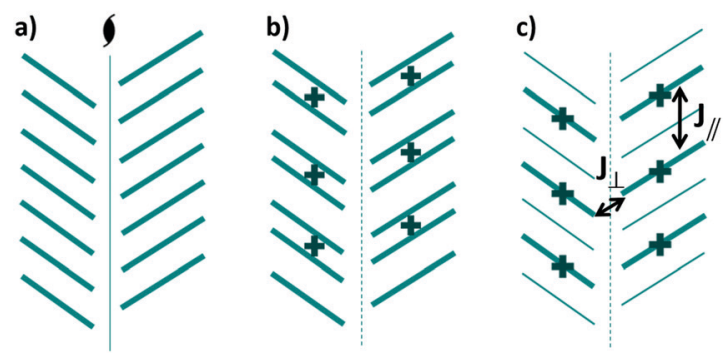

Fig. 7 Representation of a pair of donor stacks in 3; (a) connected by a screw axis and possible: bond ordering (b) and charge ordering (c) schemes for doubling of the stacking lattice parameter. Adapted with permission from ref. 39. Copyright 2015 American Chemical Society.

in nearby molecules while $J_{\|}$is an interaction between spin centers separated by neutral molecules.

In a second strategy for the search of new spin-ladder systems different thiophenic derivatives of TTF such as TTDM-TTF (thiophenothiodimethylene-tetrathiafulvalene), ETT-TTF (ethylenethiothiophene-tetrathiafulvalene) and $\alpha$-DT-TTF (alpha-dithiophenetetrathiafulvalene), were also considered as a replacement for DT-TTF in the previous compounds (Scheme 1). While a completely different structure was obtained combining TTDM-TTF with $\left[\mathrm{Au}(\mathrm{mnt})_{2}\right],{ }^{42}$ ladder structures identical to that of $\mathbf{1}$, were obtained with ETT-TTF and $\alpha$-DT-TTF. As these donors have a lower symmetry than DT-TTF, in their crystal structures, they were always found with an orientation disorder, seen as a disorder in the thiophenic $\mathrm{S}$ atom that appears randomly distributed over two positions. The consequences of this disorder are however quite different in (ETT-TTF $\left.)_{2}\left[\mathrm{Au}(\mathrm{mnt})_{2}\right]\right]^{37}(\alpha \text {-DT-TTF })_{2}\left[\mathrm{Au}(\mathrm{mnt})_{2}\right](\mathbf{4})^{43}$ and $(\alpha \text {-DT-TTF })_{2}\left[\mathrm{Au}(i \text {-mnt })_{2}\right](5)^{39}$ despite their identical crystal structures. The magnetic properties of (ETT-TTF $)_{2}\left[\mathrm{Au}(\mathrm{mnt})_{2}\right]$, do not follow the known spin-ladder behaviour but instead a Curie Weiss behaviour, ${ }^{36}$ and the electrical conductivity is about one order of magnitude lower than the DT-TTF analogue. On the other hand, 4 presents a spin-ladder behaviour comparable to the previously described in DT-TTF salts. ${ }^{43}$ The reason for the different behaviour in these salts can be understood as resulting from the different contribution of the thiophenic sulphur atom to the HOMO in ETT-TTF and $\alpha$-DT-TTF. As shown in Fig. 8 in $\alpha$-DTTTF the HOMO presents nodes over the thiophenic S atoms, at variance with ETT-TTF where these $\mathrm{S}$ atoms present a significant contribution to the HOMO. This explains why the same type of orientation disorder between neighbouring molecules can give rise to either weakly disorder or strongly disorder effects in the spin-ladder.

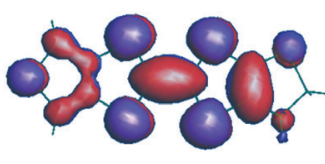

ETT-TTF

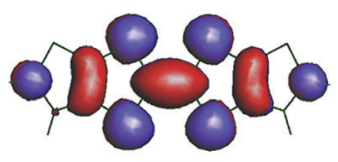

$\alpha$-DT-TTF
Fig. 8 HOMO schematic representation for ETT-TTF and $\alpha$-DT-TTF. Reproduced with permission from ref. 43. Copyright 2013 American Chemical Society. 
In the search for other related spin-ladder compounds it was also used the $\left[\mathrm{Co}(\mathrm{mnt})_{2}\right]^{-}$anion that is known to form diamagnetic dimers $\left[\mathrm{Co}(\mathrm{mnt})_{2}\right]_{2}{ }^{2-} \cdot{ }^{44,45}$ Its combination with DT-TTF failed to yield well crystallized materials, ${ }^{36}$ but by combination with $\alpha$-DTTTF it was obtained the salt $(\alpha \text {-DT-TTF })_{2}\left[\mathrm{Co}(\mathrm{mnt})_{2}\right]$ with donors arranged also in a ladder structure but of a different type as shown in Fig. 9. ${ }^{39}$ This structure is triclinic, space group $P \overline{1}$, where pairs of donor stacks, alternate with stacks of dimerized $\left[\mathrm{Co}(\mathrm{mnt})_{2}\right]$ anions. However, the donor molecules are not connected by a screw axis and present a different tilting towards the stacking axis $a$, being almost perpendicular (Fig. 9b) with a different overlap mode. The Co atom appears disordered in two closely spaced positions indicating that the structure is an average resulting from the superposition of two possible ways of forming dimers $\left[\mathrm{Co}(\mathrm{mnt})_{2}\right]_{2}{ }^{2-}$ (Fig. 9c), with a geometry comparable to that observed in an identical average structure of uncorrelated dimerized chains ${ }^{44,45}$ or isolated dimers. ${ }^{46}$ Each anion column is surrounded by six donor columns and there are 8 short donor-acceptor contacts $(\mathrm{N} \cdots \mathrm{S}$, $\mathrm{N} \cdots \mathrm{H}, \mathrm{S} \cdots \mathrm{S}$ ) between each anion. The dimerization phase of $\left[\mathrm{Co}(\mathrm{mnt})_{2}\right]_{2}{ }^{2-}$ stacks appears however uncorrelated in the $b, c$ plane.

Despite the donor ladder structure, the magnetic susceptibility of this compound does not presents a spin-ladder behaviour. This is ascribed to disorder effects induced by the uncorrelated dimerised anionic chains, which present several short contacts with the donors.

a)

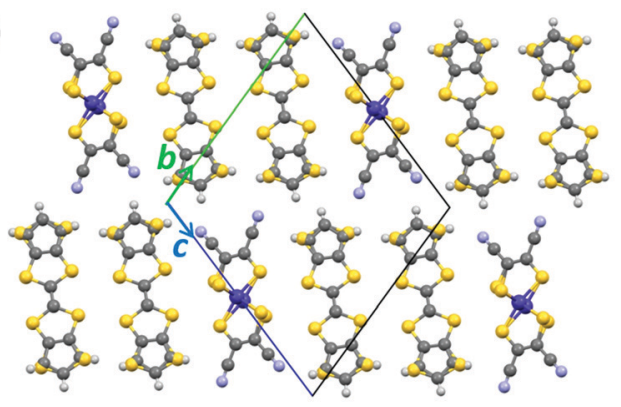

b)
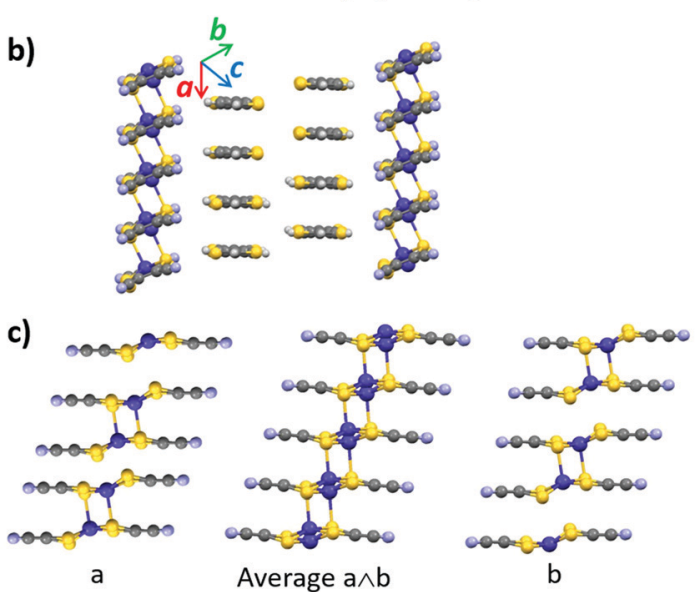

Fig. 9 Crystal structure of $(\alpha-D T-T T F)_{2}\left[\mathrm{Co}(m n t)_{2}\right]$ : (a) views along the stacking axis; (b) partial views of layers of neighbouring donor and acceptor chains along the molecular chain axis a; (c) different dimerization phases of $\left[\mathrm{Co}(\mathrm{mnt})_{2}\right]_{2}$ stacks (patterns a and b) whose overlap leads to the average structure observed. Adapted with permission from ref. 39 . Copyright 2015 American Chemical Society.
As a summary the search for new spin-ladder systems among derivatives of (DT-TTF $)_{2}\left[\mathrm{Au}(\mathrm{mnt})_{2}\right]$ was successful but limited to a very narrow range of derivatives based on very identical anions $\left(\left[\mathrm{Au}(\mathrm{mnt})_{2}\right],\left[\mathrm{Cu}(\mathrm{mnt})_{2}\right]\right.$ and $\left.\left[\mathrm{Au}(i \text {-mnt })_{2}\right]\right)$ or quite similar donors (DT-TTF and $\alpha$-DT-TTF). Some combinations afforded salts with related double chain arrangement of the donors, which however failed to display spin-ladder behaviour due to strong disorder effects, either in the donors (ETT-TTF) or in the anions $\left(\left[\mathrm{Co}(\mathrm{mnt})_{2}\right]\right)$. The salts of $\alpha$-DT-TTF provided an interesting example of a weakly disordered spin-ladder system.

The attempts to dope these spin-ladder compounds with diamagnetic anions, which could create holes in the ladders, has been so far unsuccessful, and the doping with paramagnetic anions $\left(\left[\mathrm{Ni}(\mathrm{mnt})_{2}\right]^{-}\right)$and $\left(\left[\mathrm{Pt}(\mathrm{mnt})_{2}\right]^{-}\right)$promoted the formation of completely different structures. ${ }^{47}$

\subsection{Spin-ladders based on other TTF derivatives (BEDT-TTF)}

BEDT-TTF (bis(ethylenedithio)-tetrathiafulvalene, Scheme 1) is probably the most prolific TTF derivative, giving rise to several stacked structures with interesting electronic properties in a large a diversity of ground states. The large number of sulfur atoms in the molecular periphery enables the establishment of significant intermolecular interactions through $\mathrm{S}$. .S contacts and when the donors in the solid state are partially oxidized, highly conducting systems with large bandwidths can be obtained. When the donor is fully oxidized to a radical species (BEDT-TTF) ${ }^{\bullet+}$ it carries a spin and its ionic salts as a rule are Mott insulators.

Spin-ladder behaviour based on coupled (BEDT-TTF) ${ }^{\bullet+}$ chains was reported in the salts (BEDT-TTF) $\left[\mathrm{Zn}(\mathrm{SCN})_{3}\right](6)^{48,49}$ and (BEDT-TTF) $\left[\mathrm{Co}(\mathrm{mnt})_{2}\right](7) .{ }^{50}$ In both salts the fully oxidized donor molecules (BEDT-TTF) ${ }^{\bullet}$ are arranged in similar regular chains of molecules, coupled in pairs by side $S$. . S contacts and separated by diamagnetic anions $\left[\mathrm{Zn}(\mathrm{SCN})_{3}\right]^{-}$and $\left[\mathrm{Co}(\mathrm{mnt}-)_{2}\right]^{-}$as shown in Fig. 10. Their electrical conductivity is small and thermally activated, denoting localisation due to a Mott insulating regime typical of fully ionic salts.

In the crystal structure of $\mathbf{6}$ there are four type of donor-donor interactions as shown in (Fig. 10a). Based on extended-Hückel calculation and assuming an on-site repulsion parameter $U \cong 1 \mathrm{eV}$ the authors could estimate the associated exchange interactions, labelled in (Fig. 10a). The largest one along the chains was estimated as $J_{1} / k_{\mathrm{B}}=966 \mathrm{~K}$, two interchain interactions estimated as $J_{2} / k_{\mathrm{B}}=5.2 \mathrm{~K}$ and $J_{3} / k_{\mathrm{B}}=86.4 \mathrm{~K}$, and a much weaker one between dimers $J_{4} / k_{\mathrm{B}}=0.09 \mathrm{~K}$. As the dominant magnetic interaction is in the stack direction, with interchain interactions one order of magnitude smaller, this compound should be regarded as a weakly-coupled spin-ladder $\left(J_{\|} \gg J_{\perp}\right){ }^{48}$

From the exponential increase of the susceptibility of 6 above $100 \mathrm{~K}$ using eqn (2), it could be extracted a spin-gap $\Delta /$ $k_{\mathrm{B}}=340 \mathrm{~K}$. The absence of magnetisation data in a more extended temperature range above $300 \mathrm{~K}$ did not allowed the determination of the effective ladder parameters $\left(J_{\|}, J_{\perp}\right)$, but the exponential decay of the susceptibility below $300 \mathrm{~K}$ provided for the first-time evidence for the existence of spin-gap in a situation close to the weak-coupling limit of $1 \mathrm{D}$ AF chains $\left(J_{\perp} / J_{\|} \cong 0.1\right) .{ }^{48}$ In this regard it is worth mentioning that early 

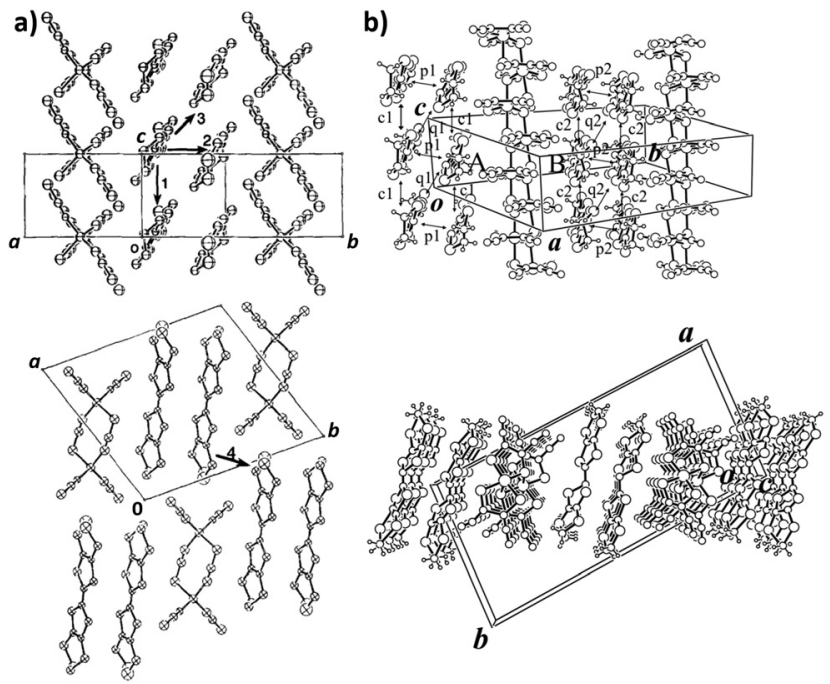

Fig. 10 Crystal structures of (BEDT-TTF) $\left[\mathrm{Zn}\left(\mathrm{SCN}_{3}\right]\right.$ (6) (a) and (BEDTTTF) $\left[\mathrm{Co}(\mathrm{mnt})_{2}\right]$ (7) (b) viewed along the molecular long axis of BEDT-TTF (top) and viewed along the stacking axis (bottom). Adapted with permission from ref. 48 and 50. Copyright 1997 Elsevier Science Ltd. Copyright 2004 Elsevier B.V.

models suggested that the spin gap would vanish bellow a critical value of $J_{\perp} / J_{\|}=0.4^{7}$ but latter it was established that it exists for all non-zero values of $J_{\perp} / J_{\|}{ }^{8}$

The crystal structure of 7 presents two crystallographic distinct pairs of donor chains (Fig. 10b), both similar to the one in (BEDT-TTF)Zn(SCN) $)_{3}$. As in the $\mathrm{Zn}$ compound there are 4 interdonor interactions for each pair of chains denoted as $c, p$ and $q$ in (Fig. 10b). As the two pairs of chains are not equivalent the intermolecular interactions are expected to be also slightly different in the two chains, but the calculated values present similar relative magnitudes, comparable to the $\mathrm{Zn}$ compound, with the largest interaction being along the chains and much smaller interchain interactions. From the exponential decrease of the susceptibility below room temperature, a spin gap of $250 \mathrm{~K}$ could be extracted by fitting to eqn (2). ${ }^{50}$

\subsection{Spin-ladders based on dithiolene complexes}

Transition metal bisdithiolene complexes are inorganic analogues of TTF, where the metal replaces the central $\mathrm{C}=\mathrm{C}$ bond. They share the same type of delocalized frontier orbitals and present different oxidation states that can be easily accessible. $\left[\mathrm{M}(\mathrm{dmit})_{2}\right]$ (Scheme 2) with several S atoms in the periphery, being able to establish multiple interactions in the solid state, is one of the most explored transition metal bisdithiolene complexes as building blocks for electronic materials. Both homoleptic and heteroleptic dithiolene complexes, based in dmit derivatives (Scheme 2), have been reported as spin carrying units in molecular spin-ladders.

3.4.1. Heteroleptic dithiolene complexes. One group of spin-ladders based on heteroleptic dithiolene complexes was found in some members of a large family of compounds described by Fourmigué et al. ${ }^{51}$ The charge transfer complexes reported with spin-ladder behaviour are based on flexible organometallic ciclopentadienil (Cp) dithiolene complexes of Mo and $\mathrm{W}$, as

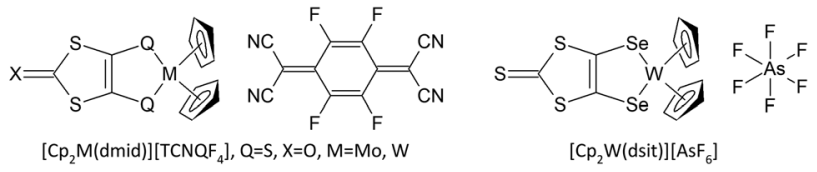

$\left[\mathrm{Cp}_{2} \mathrm{M}(\mathrm{dmit})\right]\left[\mathrm{TCNOF}_{4}\right], \mathrm{Q}=\mathrm{S}, \mathrm{X}=\mathrm{S}, \mathrm{M}=\mathrm{W}$
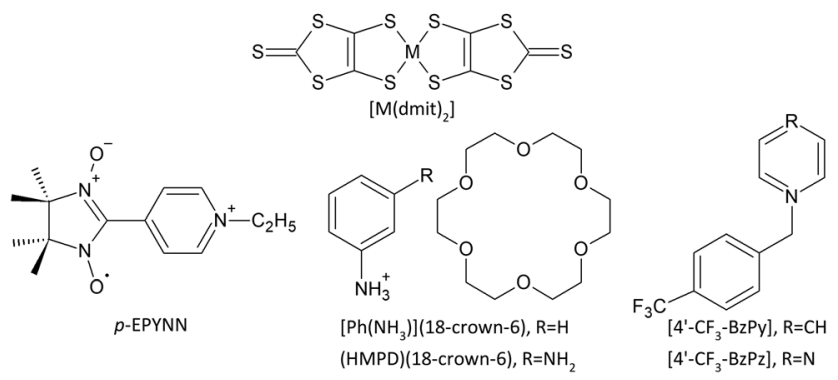

Scheme 2 Molecular building blocks of dithiolene based spin-ladders.

donors, with the strong electron acceptor $\mathrm{TCNQF}_{4}{ }^{52}$ or $\mathrm{AsF}_{6}{ }^{-}$ anions $^{53}$ (Scheme 2).

In these salts the radical anion $\mathrm{TCNQF}_{4}{ }^{\bullet-}$ is dimerised into diamagnetic units $\left[\mathrm{TCNQF}_{4}\right]_{2}{ }^{2-}$ and the paramagnetic properties are only due to the cationic complexes $\left.\left[\mathrm{Cp}_{2} \mathrm{M} \text { (dithiolene }\right)_{2}\right]^{+}$ where the unpaired electron depending on the metal, can be more or less delocalized over the entire dithiolene ligand. $\pi-\pi$ interactions between the ligands promote the formation of head to tail radical dimers in the solid state structure of these compounds, with minor variations depending on the ligand type. ${ }^{52}$

The compounds $\left[\mathrm{Cp}_{2} \mathrm{M}(\mathrm{dmid})\right]\left[\mathrm{TCNQF}_{4}\right]$ with $\mathrm{M}=\mathrm{Mo}(\mathbf{8})$ and $\mathrm{W}$ (9), are isostructural and as shown in Fig. 11 the interacting pairs of $\left[\mathrm{Cp}_{2} \mathrm{M}(\mathrm{dmid})_{2}\right]$ constitute rungs of a ladder that propagates along $c$ axis with short $S \cdots S$ contacts and the ladders are well separated by diamagnetic anionic pairs $\left[\mathrm{TCNQF}_{4}\right]_{2}{ }^{2-} \cdot{ }^{52}$ Although not isostructural the compound $\left[\mathrm{Cp}_{2} \mathrm{~W}(\mathrm{dsit})_{2}\right]\left[\mathrm{AsF}_{6}\right](\mathbf{1 1})$ presents a similar packing pattern with identical ladders. $^{53}$

The magnetic parameters obtained from the fit of eqn (2), (3) and (5) to the experimental data are listed in Table 1, characterizing these compounds as strong-rail spin-ladders. The larger coupling parameters in the $\left[\mathrm{Cp}_{2} \mathrm{Mo}(\mathrm{dmid})_{2}\right]$ complex, when compared with the isostructural $\mathrm{W}$ analogue, is a consequence of the larger ligand contribution to the SOMO.
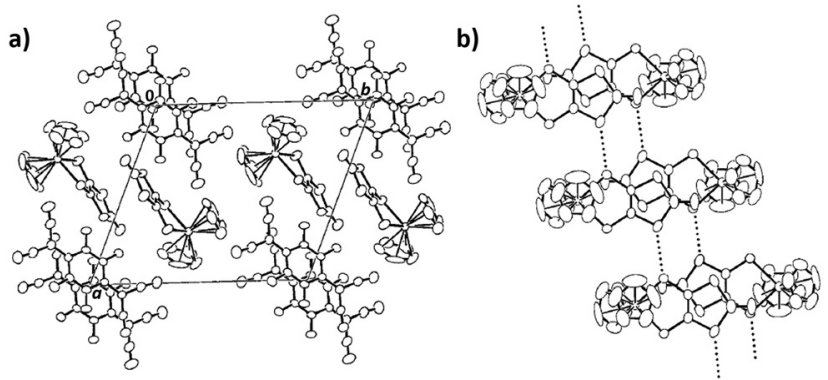

Fig. 11 Crystal structure of $\left[\mathrm{Cp}_{2} \mathrm{Mo}(\mathrm{dmid})\right]\left[\mathrm{TCNQF}_{4}\right]$ (8): (a) viewed along $C$ and (b) ladder structure formed by the $S=\frac{1}{2}$ cations. Adapted with permission from ref. 52. Copyright 1998 Wiley-VCH. 


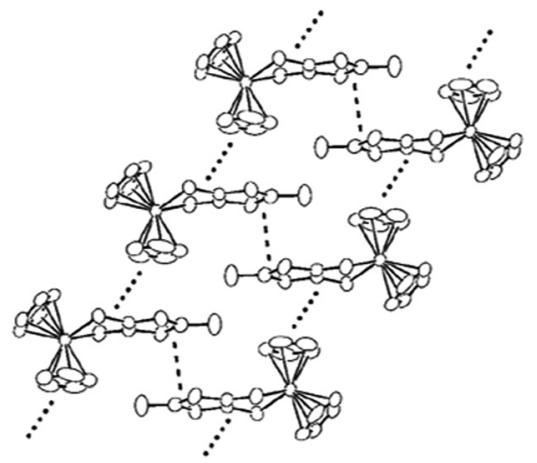

Fig. 12 Representation of the solid-state organization of the $\left[C p_{2} W(d m i t)\right]$ radical cations in $\left[\mathrm{Cp}_{2} \mathrm{~W}(\mathrm{dmit})\right]\left[\mathrm{TCNQF}_{4}\right]$ (10). Dotted lines are intra-dimer and inter-dimer interaction. Reproduced with permission from ref. 52. Copyright 1998 Wiley-VCH.

Another type of dimer association is observed in $\left[\mathrm{Cp}_{2} \mathrm{~W}(\mathrm{dmit})\right]$ $\left[\mathrm{TCNQF}_{4}\right](\mathbf{1 0})^{52}$ with the organometallic cations not in a face-toface arrangement as observed for the previous structures, but instead laterally displaced (Fig. 12), with short dmit/dmit intermolecular contacts. These dimers interact with each other only through dmit/Cp interactions along the $\mathrm{TCNQF}_{4}$ stacking axis $a$, giving rise to a slightly different ladder motif (Fig. 12). From the susceptibility data authors could deduce a spin gap value of $40 \mathrm{~K}^{52}$

3.4.2. Bisdithiolene complexes. The $\pi$-radical anion $\left[\mathrm{Ni}(\mathrm{dmit})_{2}\right]^{-}$ has been at the basis of at least four types of spin-ladder systems. Due to its capability to establish strong $\pi-\pi$ interactions, all the compounds based on this anion can be viewed as assemblies of strong dimers of $\left[\mathrm{Ni}(\mathrm{dmit})_{2}\right]^{-}$and the corresponding spin-ladders are characterised as strong rung ladders and with large spin gaps.

The first $\left[\mathrm{Ni}(\mathrm{dmit})_{2}\right]^{-}$based spin-ladder system was the compound $p$-EPYNN[Ni(dmit) $\left.)_{2}\right]$ (12) (Scheme 2) reported by Imai et al. ${ }^{55,56}$ where the cation is an organic radical species. Its crystal structure comprises two kinds of $1 \mathrm{D}$ chains parallel to $c$ axis; almost planar $p$-EPYNN ${ }^{+}$cations side-by-side and dimers of $\left[\mathrm{Ni}(\mathrm{dmit})_{2}\right]$ with an interplanar distance of $3.73 \AA$ (Fig. 13). By comparison with the analogous compound based on the diamagnetic anion $\left[\mathrm{Au}(\mathrm{dmit})_{2}\right]$ it was established ${ }^{57}$ that the $p$-EPYNN contribution to the susceptibility can be described by a model of a 1D chain with weak ferromagnetic interactions, and it was considered independent of the $\left[\mathrm{Ni}(\mathrm{dmit})_{2}\right]$ ladder contribution.

In view of the structure of complex 12 (Fig. 13) being composed of closely spaced metal over metal dimers it is expected that $J_{\perp}>J_{\|}$and indeed intermolecular transfer integrals estimated from extended Hückel calculations of the orbital overlaps indicate that $J_{\perp} / J_{\|} \approx 10^{4}$, placing this compound near a very extreme situation of isolated dimers. However, the ladder model (eqn (2)) gave a better fit than the dimer model to the $\left[\mathrm{Ni}(\mathrm{dmit})_{2}\right]$ contribution, and the authors were able to extract a spin gap of $940 \mathrm{~K}$ by a fit to eqn (2) (Fig. 14). The lack of susceptibility data at higher temperatures, near or above the spin gap, did not enable the authors to obtain values for the ladder parameters $J_{\|}$and $J_{\perp} \cdot{ }^{55}$

Compound 12 was at the basis of a study of the effect of nonmagnetic impurities in a spin-ladder by doping with diamagnetic anions. It was possible to prepare solid solutions $p$-EPYNN

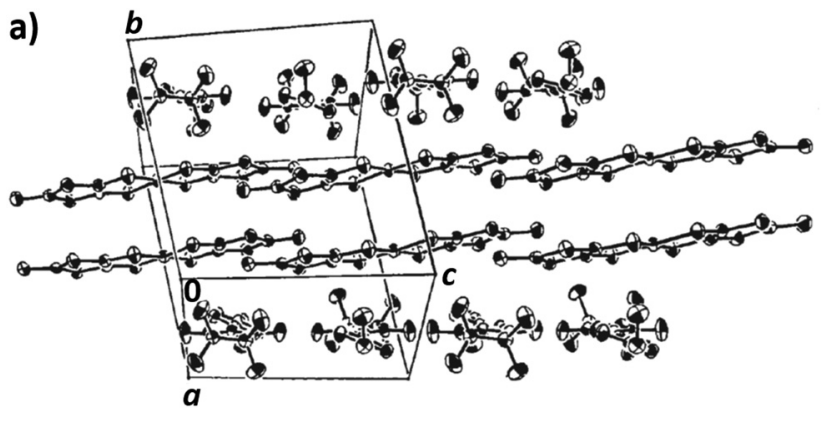

b)

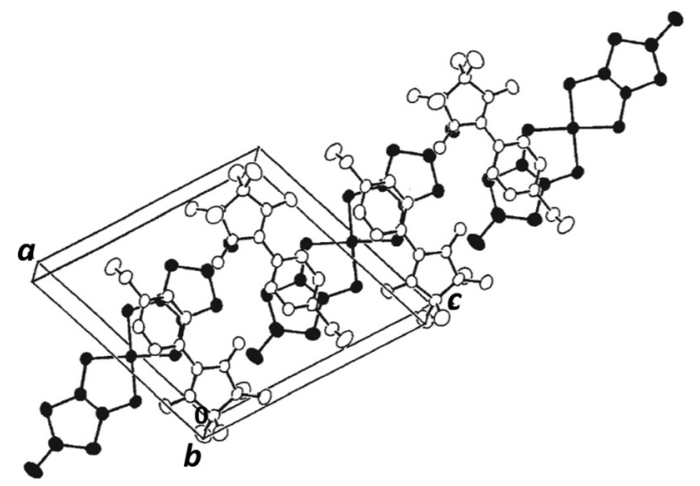

Fig. 13 Crystal structure of $p$-EPYNN[Ni(dmit) $)_{2}$ (12): (a) view along the $p$-EPYNN long axis and (b) view perpendicular to the [Ni(dmit) $)_{2}$ anions. Adapted with permission from ref. 54. Copyright 2001 The Physical Society of Japan.

$\left[\mathrm{Ni}(\mathrm{dmit})_{2}\right]_{(1-x)}\left[\mathrm{Au}(\mathrm{dmit})_{2}\right]_{x}$ with $x$ up to $0.5 .^{58}$ The magnetic susceptibility measurements show that the doping results in an increase of the Curie contribution of defects in the ladder and an enhancement of AF behaviour of $p$-EPYNN. ${ }^{54,58}$

Spin-ladder behaviour was also recently described in two isostructural compounds based on $\left[\mathrm{Ni}(\mathrm{dmit})_{2}\right]^{-}$with benzylpyridinium (13) and benzylpyrazinium (14) cations $\left(1-N-\left(4^{\prime}-\right.\right.$ trifluoromethylbenzyl) pyridinium and $1-N-\left(4^{\prime}\right.$-trifluoromethyl benzyl)pyrazinium, Scheme 2). ${ }^{59}$

These complexes crystallize in monoclinic space group $P \overline{1}$ with very similar cell parameters and packing patterns as shown in Fig. 15. The planar $\left[\mathrm{Ni}(\mathrm{dmit})_{2}\right]^{-}$monoanions form face-to-face $\pi$-type dimers, and these dimers are aligned side by side into a ladder-type arrangement via lateral-to-lateral $\mathrm{S} \cdots \mathrm{S}$ contacts along $b$ axis. $^{59}$

Compounds 13 and 14 exhibit similar magnetic properties and their magnetic susceptibility could be fitted to a Heisenberg AF two-leg spin ladder model (eqn (3)) in the high-temperature region and were reproduced well by the Troyer expression (eqn (2)) in low-temperature region. The $J_{\|}$and $J_{\perp}$ parameters obtained by fitting to experimental magnetic susceptibility data gave values of respectively $86 \mathrm{~K}, 782 \mathrm{~K}$ for 13 and $19 \mathrm{~K}, 543 \mathrm{~K}$ for 14 which by eqn (5) are indicative of gaps of 701 and $524 \mathrm{~K}$ respectively, in fair agreement with the gap values of 657 and $537 \mathrm{~K}$ obtained by fit of eqn (2) to the low temperature exponential decay of susceptibility. ${ }^{59}$

A similar spin-ladder behaviour has been also observed in other $\left[\mathrm{Ni}(\mathrm{dmit})_{2}\right]^{-}$salts (15-18) with amine ${ }^{60-63}$ or lithium $^{64,65}$ cations encapsulated by crown ethers (Scheme 2), which have 


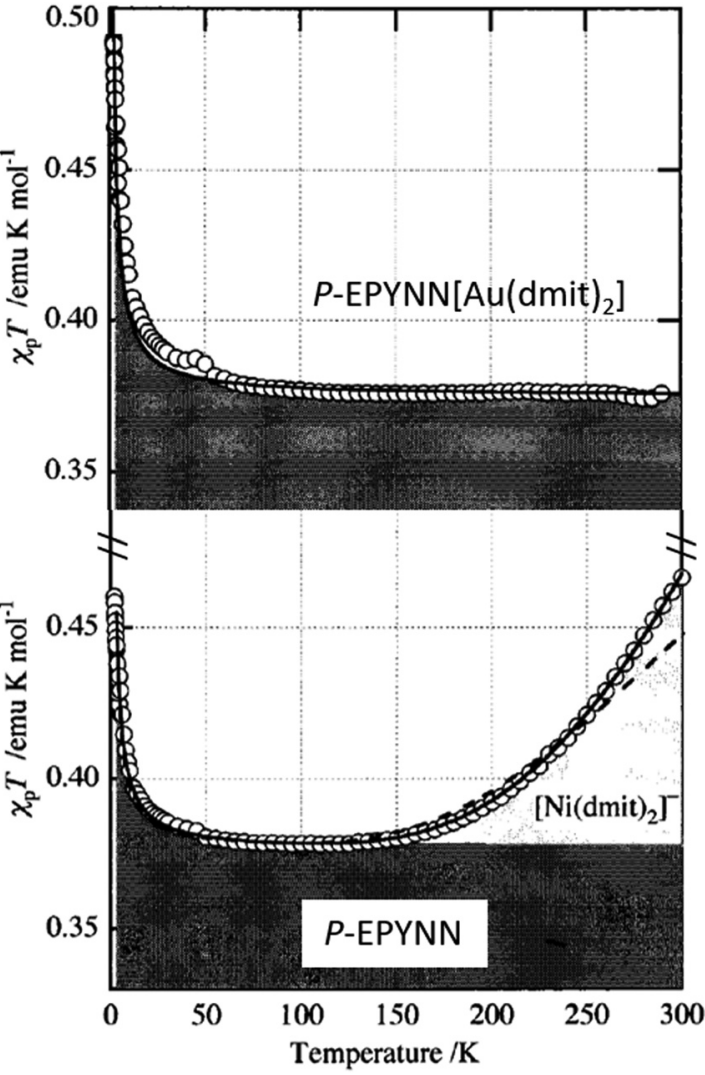

Fig. 14 Temperature dependence of the paramagnetic susceptibility $\left(\chi_{\mathrm{p}}\right)$ of $p$-EPYNN $\left[\mathrm{Au}(\mathrm{dmit})_{2}\right]$ (top) and $p$-EPYNN[Ni(dmit) $\left.)_{2}\right]$ (12) (bottom). The solid lines are the theoretical fit using equations in the text. The thick and bright regions in the bottom represent the contribution from $p$-EPYNN and $\mathrm{Ni}(\mathrm{dmit})_{2}$, respectively. The broken line in bottom is the trial best fit using a simple dimer model. Adapted with permission from ref. 57 Copyright 1999 American Chemical Society.

a)

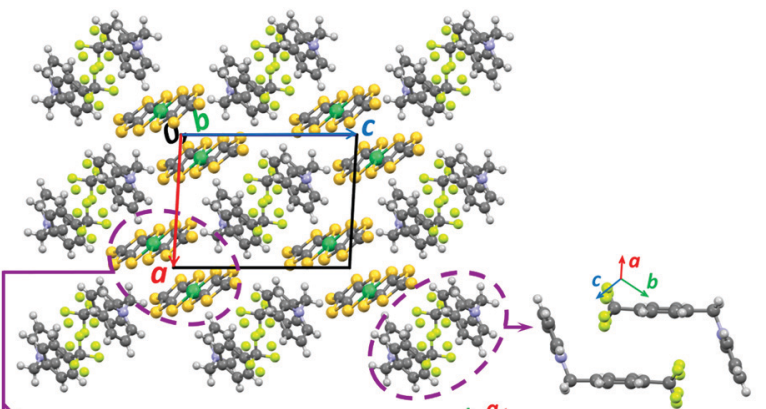

b)

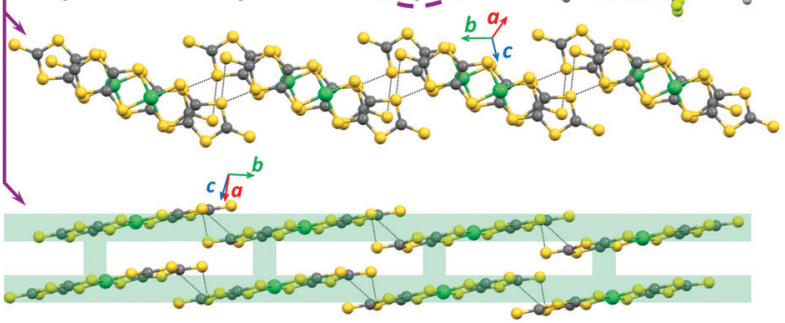

Fig. 15 Crystal structure of [4'-CF$-\mathrm{BzPy}]\left[\mathrm{Ni}(\mathrm{dmit})_{2}\right]$ (13). (a) Viewed along $b$ axis which shows a chair type cation dimer. (b) Anion chain formed via lateral-to-lateral S...S contacts and (c) anion ladder type with a representation of the ladder legs and rungs. ${ }^{59}$

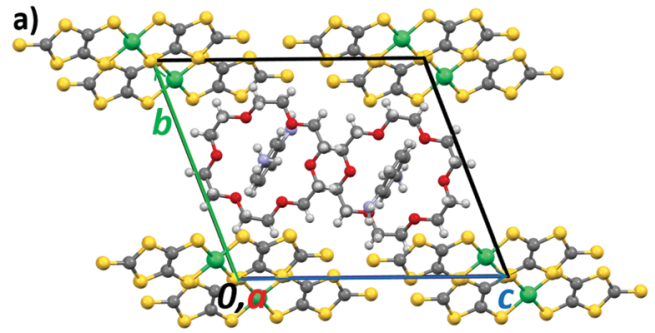

b)

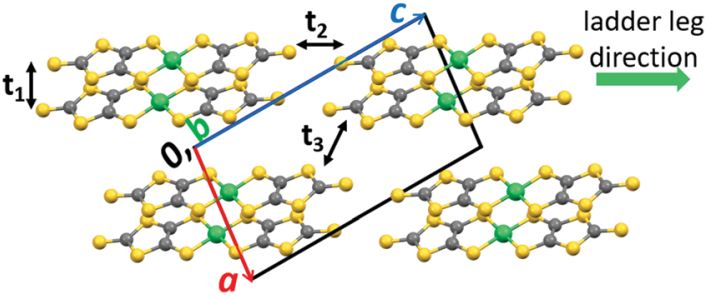

Fig. 16 Crystal structure of (HMPD)([18]crown-6)[Ni(dmit) $\left.)_{2}\right]$ (16). (a) Unit cell viewed along the $a$ axis. (b) $\left[\mathrm{Ni}(\mathrm{dmit})_{2}\right]^{-}$anion arrangement within the ac plane. Three types of intermolecular interactions $\left(t_{1}-t_{3}\right)$ were observed. ${ }^{61}$

been studied also because of the ionic conductivity associated with supramolecular cations. ${ }^{66}$

These salts present the previously mentioned type of ladder structure based in face-to-face $\pi$-type dimers of $\left[\mathrm{Ni}(\mathrm{dmit})_{2}\right]^{-}$ anions, with some minor differences. $\left[\mathrm{Ph}\left(\mathrm{NH}_{3}\right)\right](18$-crown-6) $\left[\mathrm{Ni}(\mathrm{dmit})_{2}\right](\mathbf{1 5})$ and $(\mathrm{HMPD})\left([18]\right.$ crown-6) $\left[\mathrm{Ni}\left(\mathrm{dmit}_{2}\right]\right)_{2}(\mathbf{1 6})$ are isostructural and the ladders are formed by repeating the dimers along the long axis of the anions $\left[\mathrm{Ni}(\mathrm{dmit})_{2}\right]^{-}$, the rails of the ladder being associated with contacts between terminal $\mathrm{S}$ atoms (Fig. 16).

In $\mathrm{Li}_{2}([18] \text { crown-6 })_{3}\left[\mathrm{Ni}(\mathrm{dmit})_{2}\right]_{2}\left(\mathrm{H}_{2} \mathrm{O}\right)_{4}(\mathbf{1 7})$ and $\mathrm{Li}_{2}([15] \text { crown-5 })_{3}$ $\left[\mathrm{Ni}(\mathrm{dmit})_{2}\right]_{2}\left(\mathrm{H}_{2} \mathrm{O}\right)_{2}(\mathbf{1 8})$ a slightly different pattern is observed with dimers repeating in chains by lateral S $\cdots$ S contacts (Fig. 17).

The ladder magnetic parameters obtained are listed in Table 1.

In all cases because of the large intradimer interaction, associated with face-to-face $\pi-\pi$ overlap of the anions, these compounds are characterised by strong rung interactions.
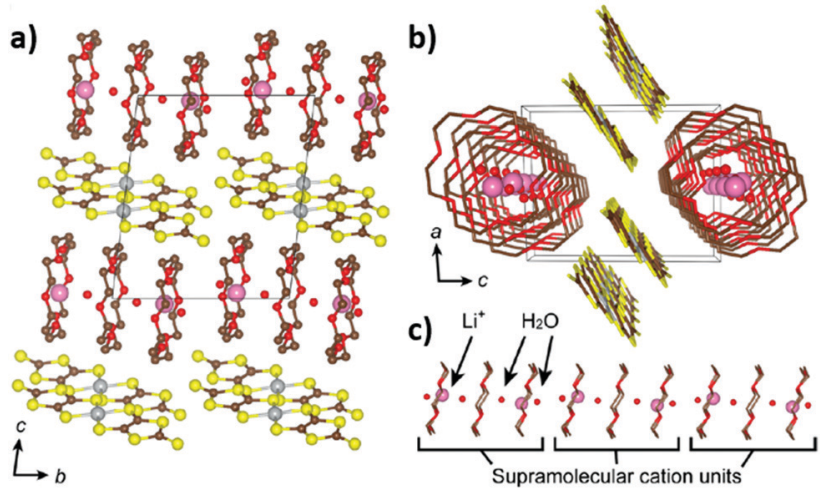

Fig. 17 Crystal structures of salt $\mathrm{Li}_{2}([18] \text { crown-6) })_{3}\left[\mathrm{Ni}(\mathrm{dmit})_{2}\right]_{2}\left(\mathrm{H}_{2} \mathrm{O}\right)_{4}(\mathbf{1 7})$ viewed along the (a) $a$ axis and (b) $b$ axis. (c) Arrangement of supramolecular cation units ([18]crown-6, $\mathrm{Li}^{+}$, and water molecules) that adopt an ion channel configuration. Reproduced with permission from ref. 64. Copyright 2018 American Chemical Society. 
In an attempt to induce ferromagnetic ordering upon doping with diamagnetic impurities, the compound $\left[\mathrm{Ph}\left(\mathrm{NH}_{3}\right)\right](18$-crown-6) $\left[\mathrm{Ni}(\mathrm{dmit})_{2}\right](\mathbf{1 5})$ was doped with the diamagnetic anions [Au(dmit $\left.)_{2}\right]$ and it was possible to prepare solid solutions $\left[\mathrm{Ph}\left(\mathrm{NH}_{3}\right)\right]$ (18-crown6) $\left[\mathrm{Ni}(\mathrm{dmit})_{2}\right]_{(1-x)}\left[\mathrm{Au}(\mathrm{dmit})_{2}\right]_{x}$ in an extended range of composition. However, no AF order was found and the properties were of a spinladder with increasing contribution of paramagnetic impurities/ defects and decrease spin-gap upon doping. ${ }^{67,68}$

\subsection{Spin-ladders based on light heteroatom radicals}

The last type of spin carrying units to be considered are neutral organic radicals based on light heteroatoms $(\mathrm{N}, \mathrm{O})$, such as nitroxyl, verdazyl and bisdithiazolyl derivatives (Scheme 3). These types of radicals are among the first building blocks employed to prepare magnetic materials based on organic molecules, namely $p$-NPNN ( $p$-nitrophenyl $\alpha$-nitronyl nitroxide) which provided the first well characterised organic crystal exhibiting bulk ferromagnetic order bellow $0.6 \mathrm{~K}^{69,70}$ or canted ferromagnets. These radicals tend to have the spin density essentially localised in heteroatoms (e.g. the nitroxide groups) and unless the SOMO is well delocalised they may present rather weak exchange interactions, often below $1 \mathrm{~K}^{71}$

The largest magnetic interactions in light heteroatom radical compounds are found in bisdithiazolyl radicals which are planar molecules with more extended and delocalised SOMOs in planar molecules.

3.5.1. Bisdithiazolyl based spin-ladders. In a series of fluorR-bisdithiazolyl radicals functionalised with long alkyl chains of different size, for $\mathrm{R}=$ butyl $(\mathrm{Bu}, 19)$, pentyl $(\mathrm{Pn}, 20)$ and hexyl $(\mathrm{Hx}, 21)$, the radicals were found be isostructural, all displaying a typical spin-ladder behaviour. ${ }^{72}$

The crystal structure of these 3 compounds, as shown in Fig. 18, consists of pairs of radical $\pi$-stacks, locked together by strong intermolecular F $\cdots \mathrm{S}$ interactions to create spin-ladder arrays.

In these compounds the rungs of the spin-ladder are associated to the slipped $\pi$-stack alignment of radicals with close $\mathrm{S} \cdot \mathrm{S}$ contacts and along the $b$ axis, separating the ladders from each other, are the long chain alkyl substituents (R). Along $a$ there are some interlader contacts associated with small interactions as discussed below.
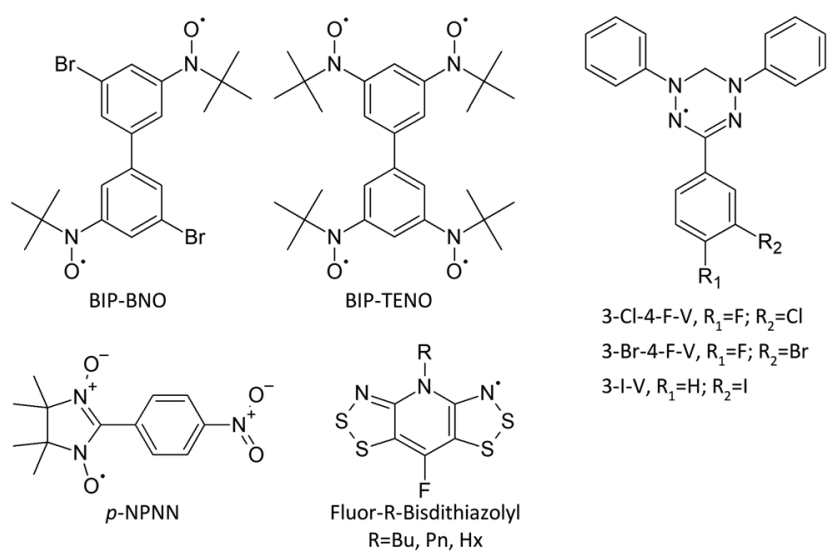

3-Cl-4-F-V, $\mathrm{R}_{1}=\mathrm{F} ; \mathrm{R}_{2}=\mathrm{Cl}$ $3-\mathrm{Br}-4-\mathrm{F}-\mathrm{V}, \mathrm{R}_{1}=\mathrm{F} ; \mathrm{R}_{2}=\mathrm{Br}$ $3-I-V, R_{1}=H ; R_{2}=I$
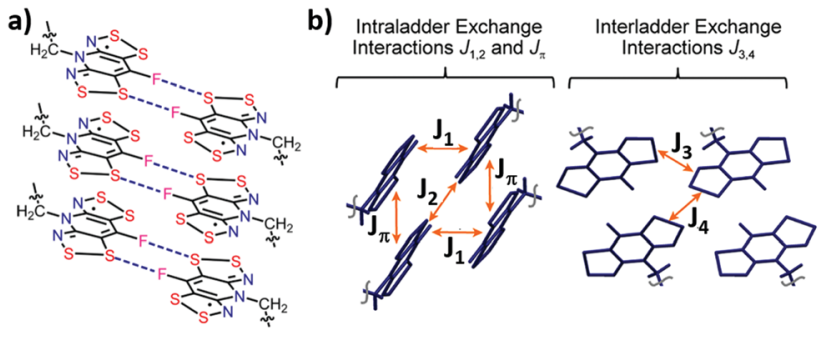

c)
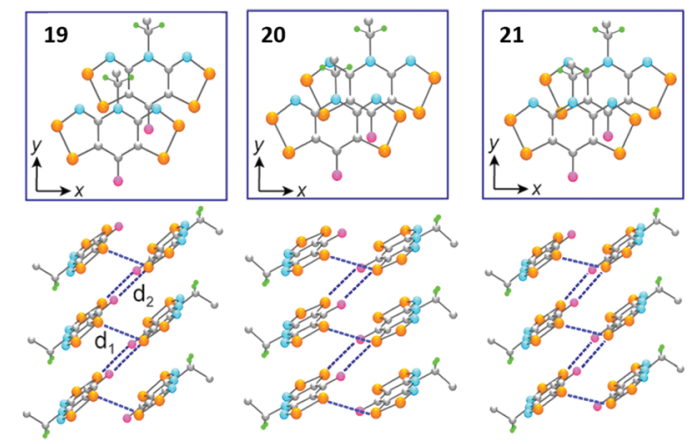

Fig. 18 (a) Bisdithiazolyl radical intermolecular S...F contacts (supramolecular synthons) that lock radicals laterally into centrosymmetic pairs. (b) Pairwise exchange interactions $J_{1}, J_{2}, J_{3}, J_{4}$, and $J_{\pi}$. (c) In the top, slippage of adjacent radicals in 19-21 along the $\pi$-stacks in local coordinates $x$ and $y$. In the bottom the ladder-like packing and contacts $d_{1}(S \ldots S)$ and $d_{2}(F \ldots S)$ connecting paired stacks. For clarity, the alkyl chains have been truncated after C2. Adapted with permission from ref. 72. Copyright 2013 American Chemical Society.

The experimental magnetic susceptibility data for these three compounds could be well fit to a strong leg spin-ladder model. This fit reveals that in 19 and 21 AF exchange interactions along the ladder legs are significantly stronger than those along the rungs, while in $\mathbf{2 0}$ they are approximately equal. The magnetic behaviour of these compounds was explored with broken-symmetry Density Functional Theory (DFT) calculations of individual pairwise exchange energies identified in Fig. 18b. ${ }^{72}$

The calculations confirmed that strong AF interactions are present in the ladder legs and rungs, with only very weak magnetic exchange between the ladders, (Table 2). The major variations observed in the exchange parameters concern mainly $J_{\pi}\left(J_{\|}\right)$, associated to small changes in the molecular overlap mode, induced by the steric constrains of the substituent $\mathrm{R}$ of different size. ${ }^{72}$

3.5.2. Nitroxyl based spin-ladders. Spin-ladder behaviour has been described also in the interesting group of organic nitroxyl polyradicals BIP-BNO (3,5'-bis( $N$-tert-butylaminoxyl)-3',5-dibromobiphenyl), (22) and BIP-TENO (3,3',5,5'-tetrakis( $N$-tert-butylaminoxyl)biphenyl), (23) (Scheme 3). ${ }^{73}$ In these polyradical molecules

Table 2 UB3LYP/6-31G(d,p) exchange parameters (K) for compounds 19-21. Negative values are ferromagnetic interactions ${ }^{72}$

\begin{tabular}{llll}
\hline Compound & $\mathbf{1 9}$ & $\mathbf{2 0}$ & $\mathbf{2 1}$ \\
\hline$J_{\pi} / k_{\mathrm{B}}$ & 48.53 & 13.09 & 45.11 \\
$J_{1} / k_{\mathrm{B}}$ & 72.47 & 66.85 & 94.80 \\
$J_{2} / k_{\mathrm{B}}$ & -0.63 & -0.12 & -0.27 \\
$J_{3} / k_{\mathrm{B}}$ & -4.45 & -1.19 & -2.03 \\
$J_{4} / k_{\mathrm{B}}$ & -4.33 & 2.09 & -2.96
\end{tabular}


the $S=1 / 2$ spin is due to unpaired electrons localized essentially in the NO groups, interacting intramolecularly through bonds as well as through space between neighbouring molecules. In 22 the two NO radicals of the molecule have an $\mathrm{AF}$ interaction across the diagonal of the molecule, while the four NO radicals in BIP-TENO have $\mathrm{AF}$ interactions between $\mathrm{NO}$ groups in opposite sides of the molecule, and ferromagnetic interaction between the two closest NO groups in the same side.

The crystal structure of 22 is monoclinic, space group $C 2 / c$, as shown in Fig. 19 and it presents chains of molecules slipstacked along $c$. It is important to note that the BIP-BNO molecule has a twofold symmetry and the two NO groups are crystallographically equivalent. The intermolecular $\mathrm{O} \cdots \mathrm{N}$ distance is $4.654(6) \AA{ }^{73}$

Thus, from the crystal structure, 22 can be considered as a two-leg spin-ladder with intramolecular rung interactions $\left(J_{\perp}\right)$ and intermolecular rail interactions $\left(J_{\|}\right)$. The temperature dependence of the paramagnetic susceptibility could be fit by a ladder model with $J_{\perp} / k_{\mathrm{B}}=72 \mathrm{~K}$ and $J_{\|} / k_{\mathrm{B}}=17 \mathrm{~K}$ and a fit of the data below $25 \mathrm{~K}$ to eqn (2) indicate an excitation gap $\Delta=47 \mathrm{~K}^{73,74}$

The crystal structure of $\mathbf{2 3}$ is orthorhombic, space group $P_{\text {bcn }}$, as shown in Fig. $20 .^{73}$ The BIP-TENO molecules have a twofold symmetry and are arranged as regular stacks along $c$ with the overlap mode shown in (Fig. 20a). The four spin centers (NO groups) in each in BIP-TENO molecule give rise to a more complex network of magnetic interactions and several intermolecular pair interactions must be considered. The tetraradical BIP-TENO is considered a dimer of the biradical BNO (1,3bis( $N$-tert-butylaminoxyl)benzene) where the two radical centers in meta position are known to be strongly ferromagnetic coupled $\left(J / k_{\mathrm{B}} \approx 600 \mathrm{~K}\right)^{75}$ and therefore a ferromagnetic interaction of
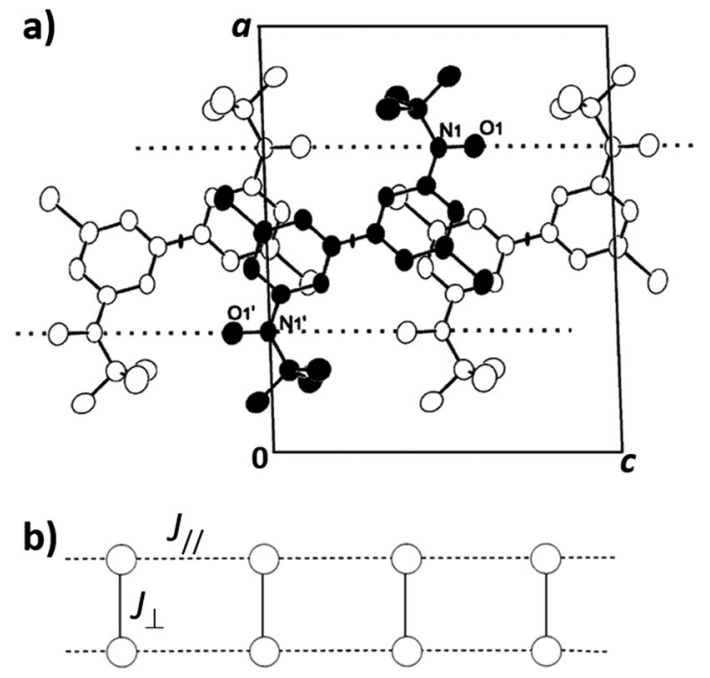

Fig. 19 (a) Crystal structure of BIP-BNO (22) viewed along the $b$ axis. The two $\mathrm{NO}$ groups $\mathrm{N}_{1} \mathrm{O}_{1}$ and $\mathrm{N}_{1}{ }^{\prime}-\mathrm{N}_{1}{ }^{\prime} \mathrm{O}_{1}^{\prime}$ are crystallographically equivalent. Dotted lines denote $\mathrm{N}$... O intermolecular contact. The tert-butyl groups are omitted for clarity. (b) Magnetic model of BIP-BNO. Open circles, $J_{\perp}$ and $J_{\|}$correspond to $S=\frac{1}{2}$ spins, intramolecular and intermolecular exchange couplings, respectively. Adapted with permission from ref. 73 . Copyright 2001 The Physical Society of Japan. a)

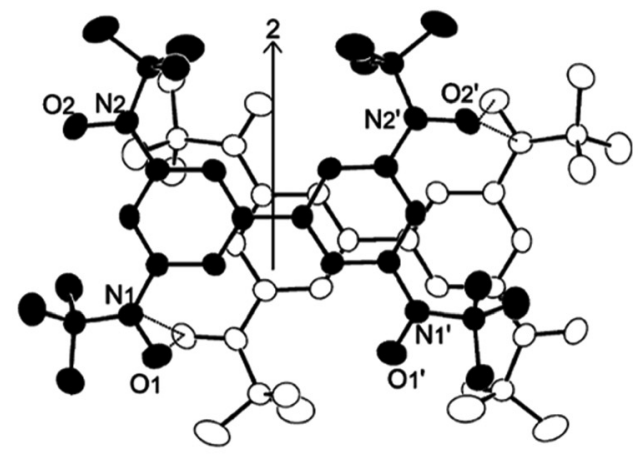

b)

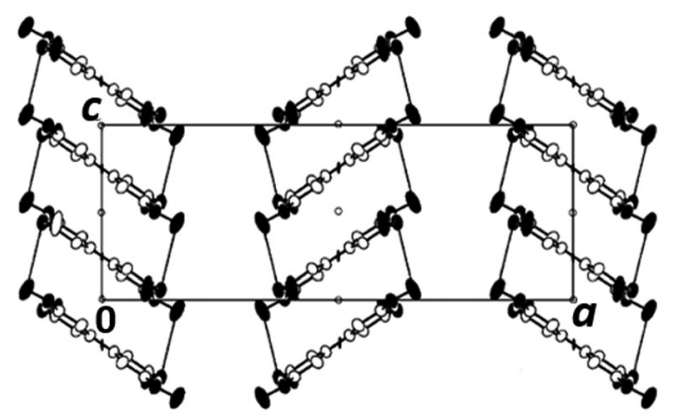

c)
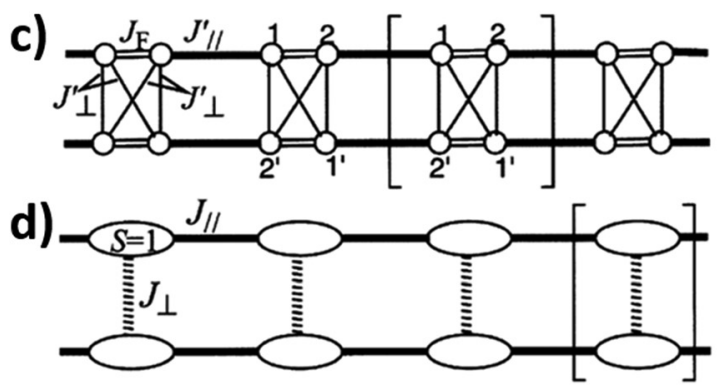

Fig. 20 (a) Overlap mode between radicals in BIP-TENO (23) with the twofold axis of the black molecule. The dotted lines correspond to the short contacts of $\mathrm{O} \cdots \mathrm{N}$ and $\mathrm{O} \cdots \mathrm{O}$. (b) Crystal structure of 23 viewed along the $b$ axis. The oxygen and nitrogen atoms of the NO groups are represented by solid ellipsoids. Thin lines correspond to the nearestneighbour contacts of $\mathrm{O} \cdots \mathrm{N}$. (c) Magnetic model of 23. A circle and a bond represent an $S=\frac{1}{2}$ and the exchange coupling, respectively $J_{F}$ and $J_{\perp}$ correspond to intermolecular exchange couplings and $J_{\|}$is the intermolecular one. (d) Simplified model in the extreme limit $J_{F} \rightarrow \infty$. Reproduced with permission from ref. 73 and 74. Copyright 2001 The Physical Society of Japan. Copyright 2002 Elsevier Science Ltd.

similar magnitude is expected between the corresponding radical centers in BIP-TENO. The other through-bond pair interactions (Fig. 20c) are expected to be AF of a smaller magnitude. In view of the large FM interaction the BIP-BNO molecule can be regarded in the temperature range of the measurements (below $300 \mathrm{~K}$ ) as a pair of AF coupled $S=1$ spins. In both BIP-BNO and BIP-TENO, the intermolecular contacts between the NO groups are responsible for AF interactions along the legs of a ladder $\left(J_{\|}\right)$.

Due to the strong ferromagnetic interaction between the spins in meta position, BIP-TENO was considered the first experimental realization of a $S=1 \mathrm{AF}$ spin-ladder, an interesting condition that has been less studied than that for $S=\frac{1}{2}$. In spite of this, it is clear that for the limiting cases of $J_{\perp}=0$ and $J_{\|}=0$, Haldane and dimer states respectively are expected. At an 
intermediate situation, a gapless transition between the Haldane and dimer systems is expected to occur. ${ }^{76,77}$ Due to the complexity of the different exchange interactions the interpretation of data is not trivial and in several aspects it still remains open to debate.

The magnetic susceptibility of $\mathbf{2 3}$ presents a broad maximum around $67 \mathrm{~K}$ but upon cooling, before approaching zero, there is a shoulder around $15 \mathrm{~K}$, better seen in the logarithmic temperature plot of $\mathrm{T}$ (inset Fig. 21b), suggesting the possible existence of two gaps in the excitation spectra. The authors analised the susceptibility data above $60 \mathrm{~K}$ considering either a four $S=\frac{1}{2}$ spin model (Fig. 20c) or a simplified $S=1$ two leg spinladder model (Fig. 20d), both reproducing data fairly well.

The parameters obtained with the $S=1$ ladder model were $J_{\|} / k_{\mathrm{B}}=50 \mathrm{~K}$ and $J_{\perp}=42 \mathrm{~K}$, while for the four spin model
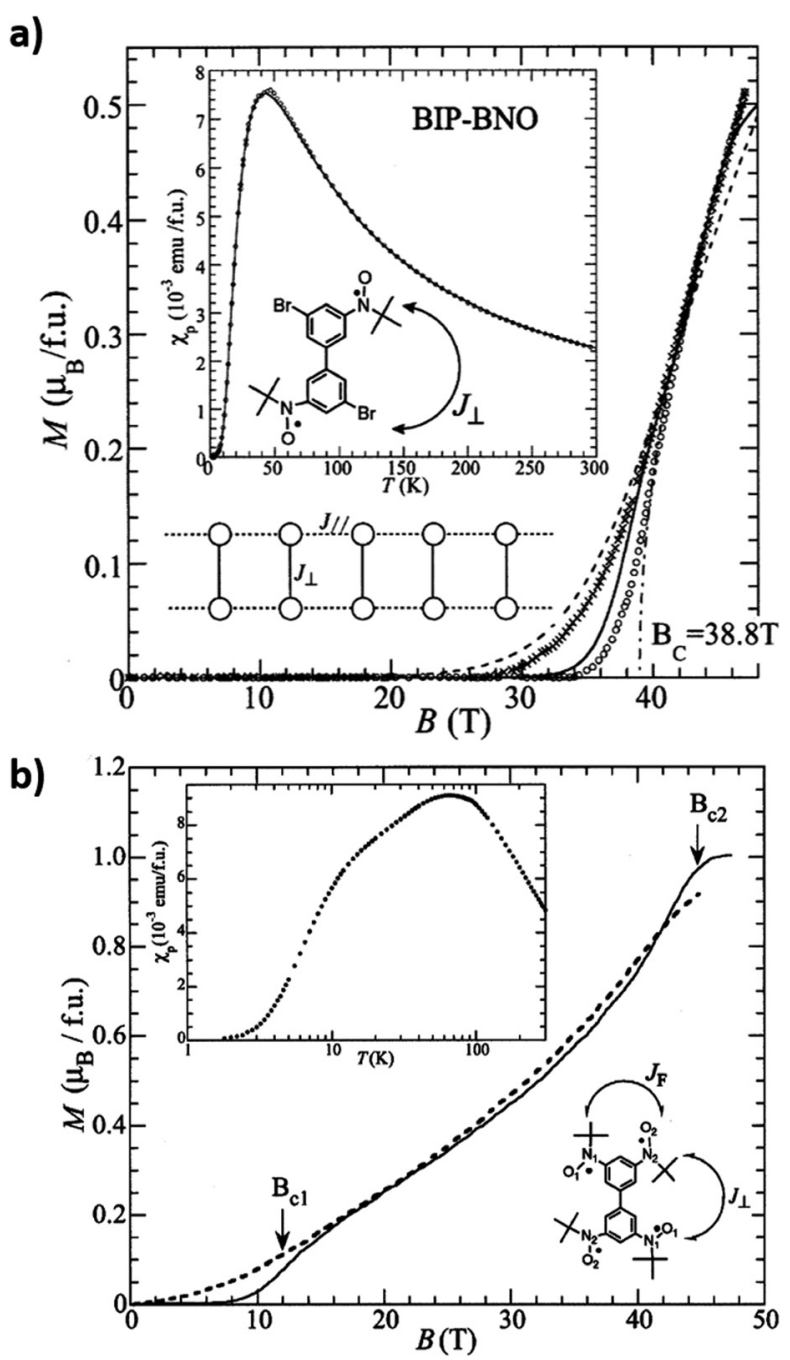

Fig. 21 (a) Magnetization curves of 22 in pulsed high magnetic fields for $1.6(\bigcirc)$ and $4.2 \mathrm{~K}(\times)$. Solid and dotted curves represent the calculations for 1.6 and $4.2 \mathrm{~K}$, respectively. Inset: Temperature dependence of the paramagnetic susceptibility. Circles are observed data after the subtraction of Curie defects (ca. 2\%). Solid curve is the calculation. (b) Magnetization curves of $\mathbf{2 3}$ in pulsed high magnetic fields. Solid and dotted curves represent the calculations for 1.7 and $4.2 \mathrm{~K}$, respectively. Inset: Temperature dependence of the paramagnetic susceptibility. Reproduced with permission from 74. Copyright 2002 Elsevier Science Ltd.
$J_{\mathrm{F}} / k_{\mathrm{B}}=590 \mathrm{~K}, J^{\prime}{ }_{\perp} / k_{\mathrm{B}}=-65 \mathrm{~K}$, and $J^{\prime} \| / k_{\mathrm{B}}=-78 \mathrm{~K}^{73}$ The magnetisation of compounds 22 and 23 were also measured at low temperatures under pulsed fields up to about $50 \mathrm{~T} .{ }^{74}$ The magnetization of 22 measured at $1.6 \mathrm{~K}$ (Fig. 21a) presents an increase above $35 \mathrm{~T}$ indicative of a critical field of $38.8 \mathrm{~T}$ which corresponds to a gap of $52 \mathrm{~K}$ in agreement with the values estimated from the analysis of the temperature dependence of the susceptibility. ${ }^{74}$ The magnetisation of 23 presents a nontrivial behaviour. While below $9 \mathrm{~T}$ the magnetisation is almost zero, indicative of a singlet ground state, upon increasing field it presents a first increase corresponding to a critical field $B_{\mathrm{c} 1}=11.6 \mathrm{~T}$ and at higher fields reaching a plateau at $B_{\mathrm{c} 2}=$ 44.8 $\mathrm{T}$ with value corresponding to $\frac{1}{4}$ of the saturation. This plateau was confirmed in measurements extended up to $70 \mathrm{~T}^{78}$ Its exact origin remains puzzling, but the plateau can be reproduced by a more complex model considering third neighbour interactions between the different spin centres. ${ }^{79-81}$

3.5.3. Verdazyl based spin-ladders. Verdazyl radicals are known for more than 5 decades but only recently started to be structurally characterised as crystalline materials and explored as components in magnetic materials. A diversity of interesting magnetic properties have been recently reported in verdazyl radicals, ${ }^{82,83}$ including spin-ladder behaviour in the series of closely related halogen substituted verdazyl derivatives 3-Cl-4-F$\mathrm{V}(24),{ }^{84} 3-\mathrm{I}-\mathrm{V}(25)$ and $3-\mathrm{Br}-4-\mathrm{F}-\mathrm{V}(26) .{ }^{85}$ A common distinctive feature of these compounds as spin-ladders is the ferromagnetic nature of the rail interactions constituting the first experimental realisations of a ladder with ferromagnetic legs, a situation that from a theoretical point of view was previously intensively discussed and well understood. ${ }^{86-91}$

As compounds 24-26 are isomorphous, their study offers a valuable opportunity to clearly put into evidence the effects of significant magnetic interactions variations, induced by small structural modifications upon substitution with different halogens $(\mathrm{F}, \mathrm{Cl}, \mathrm{Br}$ and $\mathrm{I})$ in several positions in the phenyl rings.

The structures of these salts as shown in Fig. 22a and b for $3-\mathrm{I}-\mathrm{V}$ (25), are monoclinic $P 2_{1} / n$ and consist in pairs of regular stacks along the $a$ axis. In spite of the molecules not being exactly planar, with phenyl rings being slightly rotated relatively to the central verdazyl core, they stack along the $a$ axis at short intermolecular C...C distances $d_{1}, d_{2}$ and $d_{3}$ in the range

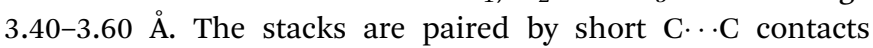
( $d_{4}$ in Fig. 22b) between one of the unsubstituted phenyl rings at distances in the range 3.67-3.75 A. Depending on the substituents, differences in the geometrical parameters are induced. The main variations are in the dihedral angle of the substituted phenyl ring while changes in the overlap mode are minor as shown in (Fig. 22d). ${ }^{84,85}$

Molecular orbital calculations show that the central verdazyl ring carries most of the spin density (63\% in 24 ), while the unsubstituted phenyl rings carry $\approx 17 \%$ each and the ring substituted with halogens carries only $\approx 7 \%$. The calculation of the intermolecular magnetic interactions between pairs of neighbouring molecules reveal two dominating ones corresponding, as expected from the structure, to the intra- and interchain interactions, evaluated as ferromagnetic and $\mathrm{AF}$ respectively with values 
a)

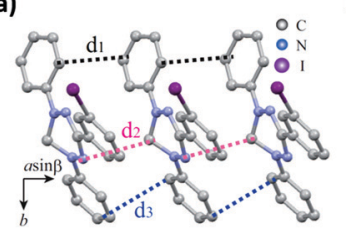

b)

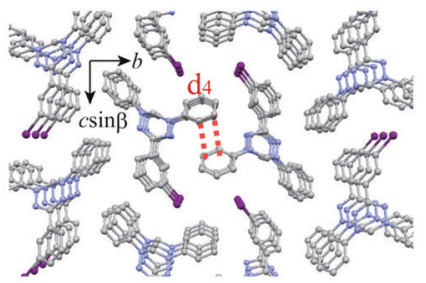

d)

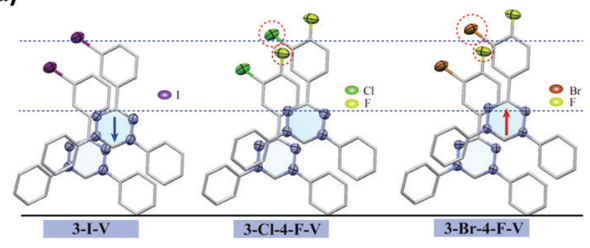

Fig. 22 Molecular arrangement of 3-I-V (25) viewed along the $c$ (a) and a axis (b). (c) Crystal structure of 3-Br-4-F-V (26) forming the spin ladder Solid and dashed lines stand for $J_{\text {rung }}$ and $J_{\text {leg, }}$ respectively. (d) Neighbouring molecules along the leg direction viewed in the direction perpendicular to the verdazyl rings. Hydrogen atoms are omitted for clarity. Broken circles enclose halogens with relatively strong electrostatic repulsion. The arrows indicate the directions of shifts in comparison to the molecular overlap for 3-Cl-4-F-V (24). Reproduced with permission from ref. 85 and 92. Copyright 2014 American Physical Society. Copyright 2014 The Physical Society of Japan.

listed in Table 3. These values predict the formation of a $S=\frac{1}{2}$ two leg ladder with ferromagnetic leg interactions, with a strong-leg character for $\mathbf{2 4}$, a strong-rung character for $\mathbf{2 6}$ and a closer to ideal situation for $25 .{ }^{84,85}$

The temperature dependence of the magnetic susceptibility (Fig. 23a) denotes for all compounds the existence of both ferromagnetic and AF interactions. Using QMC simulations the authors were able to fit the model of a $S=\frac{1}{2}$ two-leg spin-ladder with ferromagnetic interactions to the experimental data with the $J_{\|}$and $J_{\perp}$ parameters listed also in Tables 1 and $3 .^{84,85}$ These parameters are in qualitative agreement with those based on molecular orbital calculations, and were also able to reproduce fairly well the magnetization curves at $0.5 \mathrm{~K}$ (Fig. 22b), showing an excitation gap of $c a 4.5 \mathrm{~T}$ for 26 while for 24 and 25 the gap is almost vanished. The specific heath measurements under zero magnetic field (Fig. 22c) show clearly for $\mathbf{2 4}$ a $\lambda$-type anomaly indicative of a magnetic ordering transition at $T_{\mathrm{N}}=$ $1.1 \mathrm{~K}$ ascribed to interladder interactions, more efficient in promoting a 3D ordering when the expected gap is small.

Due to the relatively small coupling constants in these verdazyl derivatives the magnetization reaches saturation at easily accessible magnetic fields, below $6 \mathrm{~T}$ in compounds $\mathbf{2 4}$ and 25 and at circa $9 \mathrm{~T}$ in 26 (Fig. 23b). Further studies at low

Table 3 Experimentally estimated and calculated magnetic parameters and observed phase transition temperatures of compounds 24, 25 and $26^{84,85}$

\begin{tabular}{|c|c|c|c|c|c|c|c|c|}
\hline \multirow[b]{2}{*}{ Compound } & \multicolumn{2}{|c|}{$\left|J_{\perp} / J_{\|}\right|$} & \multicolumn{2}{|c|}{$J_{\perp} / k_{\mathrm{B}}(\mathrm{K})$} & \multicolumn{2}{|c|}{$\underline{J_{\|} / k_{\mathrm{B}}(\mathrm{K})}$} & \multirow{2}{*}{$\begin{array}{l}\text { Expected } \\
\text { gap (K) }\end{array}$} & \multirow[b]{2}{*}{$T_{\mathrm{N}}(\mathrm{K})$} \\
\hline & Est. & Calc. & Est. & Calc. & Est. & Calc. & & \\
\hline & 0.55 & 0.57 & 7.3 & 4.7 & -13.3 & -9.2 & 1.1 & 1.1 \\
\hline 25 & 0.5 & 0.53 & 5.8 & 7.8 & -11.6 & -9.4 & 0.5 & 1.4 \\
\hline 26 & 1.5 & 1.75 & 12.5 & 8.4 & -8.3 & -4.8 & 6.9 & - \\
\hline
\end{tabular}
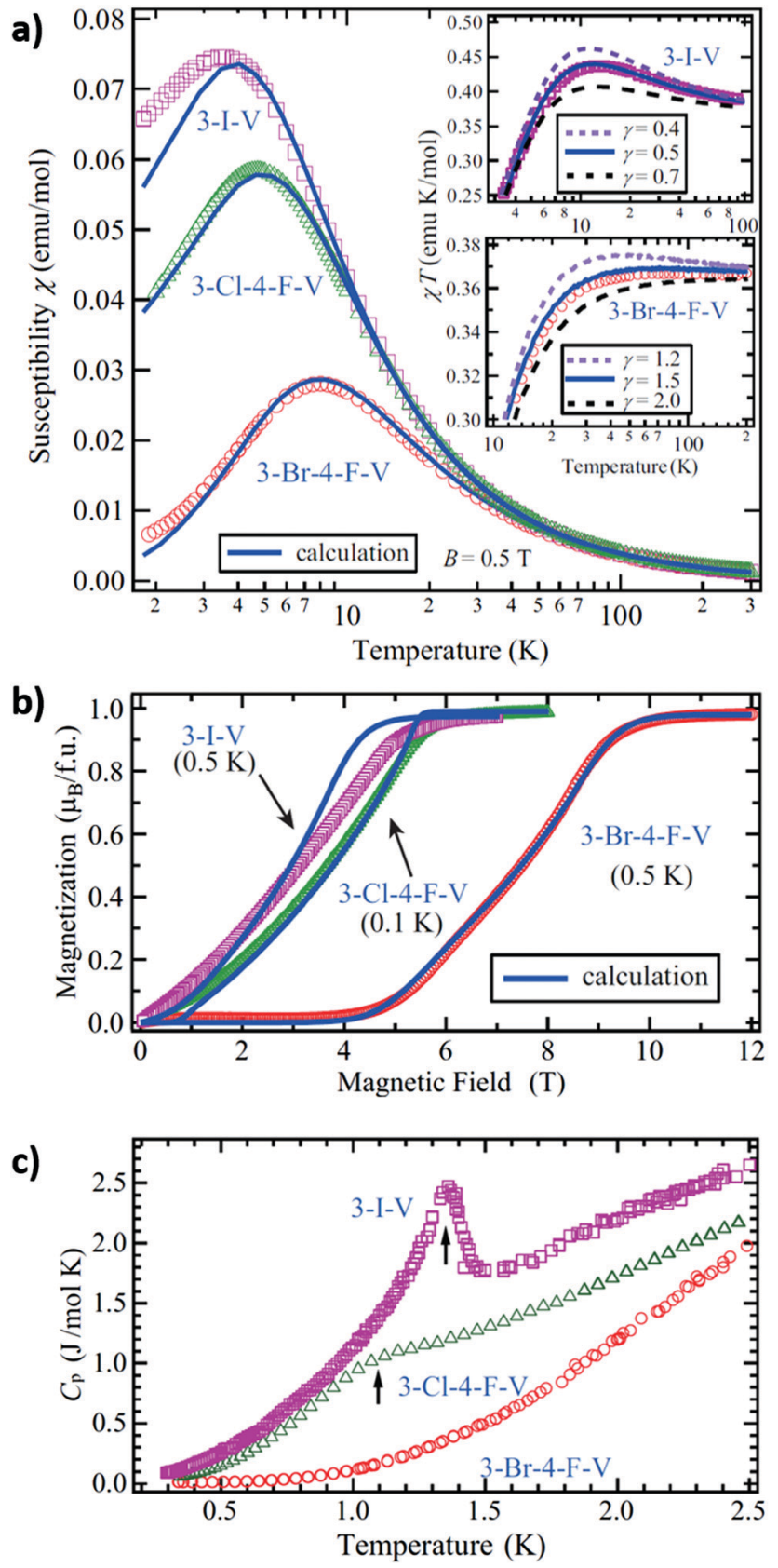

Fig. 23 (a) Temperature dependence of magnetic susceptibility $(M / H)$ at $0.5 \mathrm{~T}$ for 3-Cl-4-F-V (24), 3-I-V (25) and 3-Br-4-F-V (26). The upper and lower insets show temperature dependence of $\chi T$. (b) Magnetization curves of $\mathbf{2 4}$ at $0.1 \mathrm{~K}$ and $\mathbf{2 5}$ and $\mathbf{2 6}$ at $0.5 \mathrm{~K}$. The solid lines for $\mathbf{2 4 , 2 5}$ and 26 represent the calculated results for $\gamma=\left|J_{\text {rung }} / J_{\text {leg }}\right|=0.5,0.55$, and 1.5 , respectively. (c) Temperature dependence of specific heat at the zero field for 24, $\mathbf{2 5}$ and 26. The arrows indicate phase transition temperatures. Reproduced with permission from ref. 85. Copyright 2014 The Physical Society of Japan

temperatures down to the $\mathrm{mK}$ region and under magnetic fields, using a combination of magnetization, susceptibility and specific heat measurements, enabled to explore in detail the magnetic field versus temperature phase diagram of these compounds. These measurements reveal interesting phase diagrams with a series of phases as shown in Fig. 24. 
For $\mathbf{2 4}$ the ground state is situated very close to the quantum critical point, and successive field-induced phase transition were found, possibly originated from the interplay of low dimensionality and frustration. ${ }^{84}$

In 25, exhibiting at low field a 3D order, it is observed an additional nontrivial phase near the saturation field. ${ }^{93}$ The absence of successive phase transition with decreasing temperature and the anomalous behaviour of the magnetization curve near the

a)

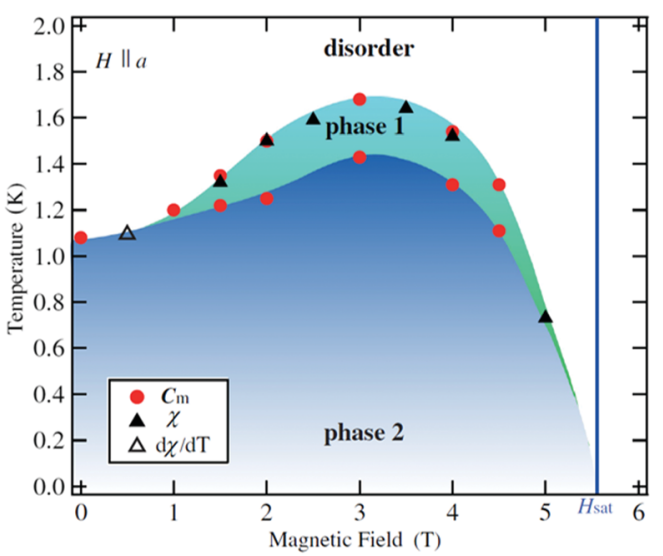

b)

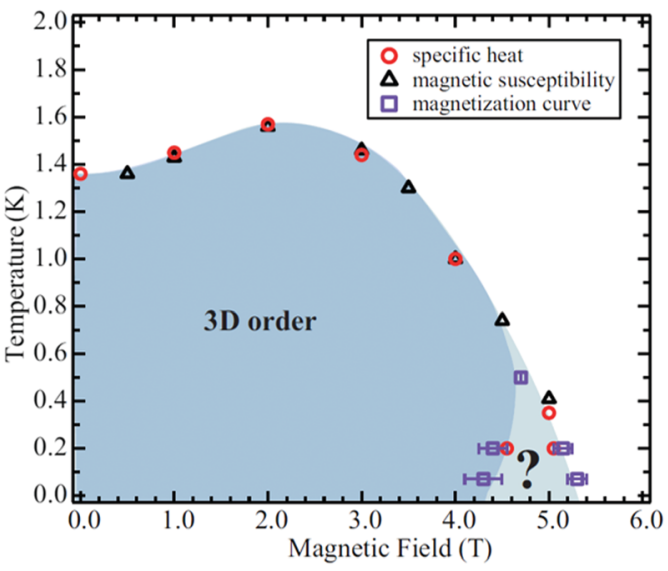

c)

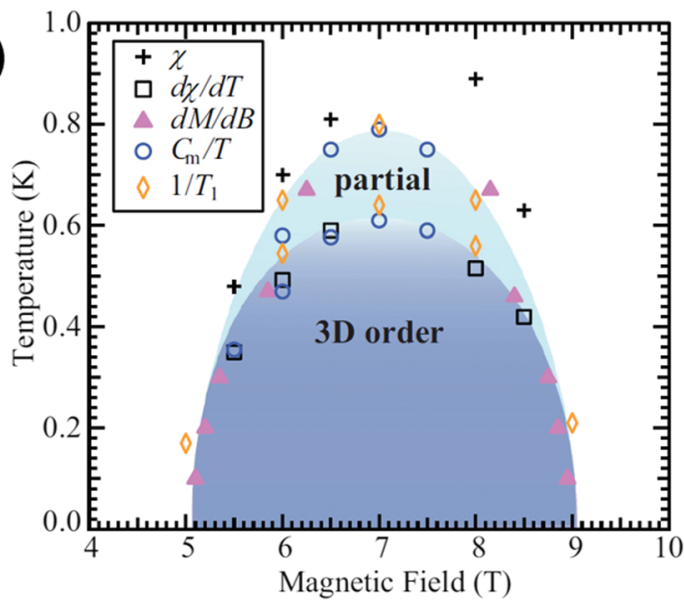

Fig. 24 Magnetic field versus temperature phase diagrams of different compounds: (a) 3-Cl-4-F-V (24), (b) 3-I-V (25) and (c) 3-Br-4-F-V (26). Reproduced with permission from ref. 84, 92 and 93. Copyright 2013, 2015 and 2014 American Physical Society.

saturation field suggest that these two phases are longitudinal spin-density-wave and spin-multipolar orders, respectively.

In 26, characterised by a strong-rung regime, successive field induced phase transitions are observed. NMR spectra suggest, for the low and high temperature phases, a partial magnetic order and incommensurate long range order respectively. ${ }^{92}$

\section{Conclusions and future prospects}

The compounds that were described in this review illustrate how developments in molecular chemistry combined with principles of crystal engineering have allowed during the last two decades the preparation of several new molecular spin-ladders, based on different type of molecules and presenting a wide range of magnetic interactions and spin values. Some of these systems constituted the experimental realisation of situations previously discussed only from a theoretical point of view, such as the $S=1$ ladders, and ladders with ferromagnetic rung interactions. The preparation of these new systems made possible physical studies of fundamental importance for the experimental testing of theoretical models relevant for understanding the behaviour of low dimensional quantum magnetic systems. The potential for further development of new spin-ladders based on molecules is large and still far from fully explored. In spite of difficulties mainly associated with the difficulties in predicting and control the crystal structures, further developments in this field of molecular magnetic systems are expected in the future.

There are two important challenges that remain yet to be successfully addressed in molecule based spin-ladder systems. One is the realization of ladders with odd or with an increasing and controlled number of legs. In principle, this can be achieved either by controlling the coupling of a finite number of chains, or by assembling in chains a finite number of linked molecular spin centres. Another challenge is the hole doping of spin-ladders, which is predicted to lead to superconductivity and may open new perspectives for the development of superconducting materials. A proper strategy for hole doping in molecular systems without disrupting the molecular structure still needs to be developed. The possible doping of the ladders in a field effect transistor structure, allowing a fine-tuning of carrier concentration by control of the gate voltage, is an attractive alternative that so far has not been successful.

\section{Abbreviations}

\section{$\alpha$-DT-TTF \\ $\left[4^{\prime}-\mathrm{CF}_{3}-\mathrm{BzPy}\right]$ \\ $\left[4^{\prime}-\mathrm{CF}_{3}-\mathrm{BzPz}\right]$ \\ 1D \\ $2 \mathrm{D}$ \\ $3-\mathrm{Br}-4-\mathrm{F}-\mathrm{V}$}

3-Cl-4-F-V

$3-\mathrm{I}-\mathrm{V}$
Alpha-dithiophene-tetrathiafulvalene

1- $N$-(4'-trifluoromethylbenzyl)pyridinium $1-N$-(4'-trifluoromethylbenzyl)pyrazinium One dimensional Two dimensional 3-(3-bromo-4-fluorophenyl)-1,5-diphenylverdazyl

3-(3-chloro-4-fluorophenyl)-1,5-diphenylverdazyl

3-(3-iodophenyl)-1,5-diphenylverdazyl 


\begin{tabular}{|c|c|}
\hline $3 \mathrm{D}$ & Three dimensional \\
\hline $\mathrm{AF}$ & Antiferromagnetic \\
\hline BEDO & Bis(ethylenedioxo)tetrathiafulvalene \\
\hline BEDT-TTF & Bis(ethylenedithio) tetrathiafulvalene \\
\hline BIP-BNO & $\begin{array}{l}\text { 3,5'-bis( } N \text {-tert-butylaminoxyl)-3', } \\
\text { 5-dibromobiphenyl }\end{array}$ \\
\hline BIP-TENO & $\begin{array}{l}3, \quad 3^{\prime}, 5,5^{\prime} \text {-tetrakis }(N \text {-tert-butylaminoxyl)- } \\
\text { biphenyl }\end{array}$ \\
\hline $\mathrm{Cp}$ & Cyclopentadienyl \\
\hline dmid & 2-Oxo-1,3-dithiole-4,5-dithiolate \\
\hline mit & 2-Thioxo-1,3-dithiole-4,5-dithiolate \\
\hline sit & 2-Thioxo-1,3-dithiole-4,5-diselenolate \\
\hline DT-TTF & Dithiophenetetrathiafulvalene \\
\hline ETT-TTF & Ethylenethiothiophenetetrathiafulvalene \\
\hline F-Bu-bisdithiazolyl & $\begin{array}{l}\text { 8-Fluoro-4-butyl-4H-bis }([1,2,3] \text { dithiazolo })- \\
{\left[4,5-b: 5^{\prime}, 4^{\prime}-e\right] \text { pyridin-2-y }}\end{array}$ \\
\hline F-Hx-bisdithiazolyl & $\begin{array}{l}\text { 8-Fluoro-4-hexyl-4H-bis([1,2,3]dithiazolo)- } \\
{\left[4,5-b: 5^{\prime}, 4^{\prime}-e\right] \text { pyridin-2-yl }}\end{array}$ \\
\hline F-Pn-bisdithiazolyl & $\begin{array}{l}\text { 8-Fluoro-4-pentyl- } 4 H \text {-bis }([1,2,3] \text { dithiazolo })- \\
{\left[4,5-b: 5^{\prime}, 4^{\prime}-e\right] \text { pyridin-2-yl }}\end{array}$ \\
\hline HOMO & Highest occupied molecular orbital \\
\hline HMPD & m-Aminoanilinium \\
\hline NMR & Nuclear magnetic resonance \\
\hline mnt & Maleonitriledithiolate \\
\hline$p$-EPYNN & $p$ - $N$-Ethylpyridinium $\alpha$-nitronyl nitroxide \\
\hline$p$-NPNN & $p$-Nitrophenyl $\alpha$-nitronyl nitroxide \\
\hline QMC & Quantum Monte Carlo \\
\hline SOMO & Single occupied molecular orbitals \\
\hline $\mathrm{TCNQF}_{4}$ & Tetrafluorotetracyanoquinodimethane \\
\hline TTDM-TTF & $\begin{array}{l}\text { Thiophenothiodimethylene- } \\
\text { tetrathiafulvalene }\end{array}$ \\
\hline SR & Muon spin resonance \\
\hline
\end{tabular}

There are no conflicts to declare.

\section{Acknowledgements}

The continuous collaboration during the past two decades with different co-authors which contributed to this topic, particularly with Prof. Concepció Rovira and Jaume Veciana is gratefully acknowledged. Work partially supported by FCT (Portugal) through research contracts UIDB/04349/2020 and LISBOA-010145-FEDER-029666.

\section{References}

1 E. Dagotto and T. M. Rice, Science, 1996, 271, 618-623.

2 D. J. Scalapino, Nature, 1995, 377, 12-13.

3 Z. Hiroi and M. Takano, Nature, 1995, 337, 41-43.

4 M. Azuma, Z. Hiroi, M. Tanako, K. Ishida and Y. Kitaoka, Phys. Rev. Lett., 1994, 73, 3463-3466.

5 F. D. M. Haldane, Phys. Rev. Lett., 1983, 50, 1153-1156.

6 M. Troyer and M. Zhitomirsky, Phys. Rev. B: Condens. Matter Mater. Phys., 1997, 55, R6117-R6120.
7 E. Dagotto, J. Riera and D. Scalapino, Phys. Rev. B: Condens. Matter Mater. Phys., 1992, 45, 5744-5747.

8 T. Barnes, E. Dagotto, J. Riera and E. S. Swanson, Phys. Rev. B: Condens. Matter Mater. Phys., 1993, 47, 3196-3203.

9 E. Dagotto, Rev. Mod. Phys., 1994, 66, 763-840.

10 T. Barnes and J. Riera, Phys. Rev. B: Condens. Matter Mater. Phys., 1994, 50, 6817-6822.

11 M. Troyer, H. Tsunetsugu and D. Würtz, Phys. Rev. B: Condens. Matter Mater. Phys., 1994, 50, 13515-13527.

12 M. H. Whangbo, Acc. Chem. Res., 1983, 16, 95-101.

13 S. Wessel, M. Olshanii and S. Haas, Phys. Rev. Lett., 2001, 87, 206407.

14 T. Giamarchi and A. M. Tsvelik, Phys. Rev. B: Condens. Matter Mater. Phys., 1999, 59, 11398-11407.

15 S. Wessel and S. Haas, Eur. Phys. J. B, 2000, 16, 393-396.

16 S. Wessel and S. Haas, Phys. Rev. B: Condens. Matter Mater. Phys., 2000, 62, 316-323.

17 Ch. Rüegg, K. Kiefer, B. Thielemann, D. F. McMorrow, V. Zapf, B. Normand, M. B. Zvonarev, P. Bouillot, C. Kollath, T. Giamarchi, S. Capponi, D. Poilblanc, D. Biner and K. W. Krämer, Phys. Rev. Lett., 2008, 101, 247202.

18 D. C. Johnston, J. W. Johnson, D. P. Goshom and A. P. Jacobson, Phys. Rev. B: Condens. Matter Mater. Phys., 1987, 35, 219-222.

19 A. W. Garret, S. E. Nagler, D. A. Tennant, B. C. Sales and T. Barnes, Phys. Rev. Lett., 1997, 79, 745-748.

20 G. Chaboussant, M.-H. Julien, Y. Fagot-Revurat, L. P. Lévy, C. Berthier, M. Horvatié and O. Piovesana, Phys. Rev. Lett., 1997, 79, 925-928.

21 T. Matsumoto, Y. Miyazaki, A. S. Albrecht, C. P. Landee, M. M. Turnbull and M. Sorai, J. Phys. Chem. B, 2000, 43, 9993-10000.

22 B. C. Watson, V. N. Kotov, M. W. Meisel, D. W. Hall, G. E. Granroth, W. T. Montfrooij, S. E. Nagnler, D. A. Jensen, R. Backov, M. A. Petruska, G. E. Fanucci and D. R. Talham, Phys. Rev. Lett., 2001, 86, 5168-5171.

23 F. M. Woodward, A. S. Albrecht, C. M. Wynn, C. P. Landee and M. M. Turnbull, Phys. Rev. B: Condens. Matter Mater. Phys., 2002, 65, 144412.

24 R. Kadono, H. Okajima, A. Yamashita, K. Ishii, T. Yokoo, J. Akimitsu, N. Kobayashi, Z. Hiroi, M. Takano and K. Nagamine, Phys. Rev. B: Condens. Matter Mater. Phys., 1996, 54, R9628(R).

25 S. Matsumoto, Y. Kitaoka, K. Ishida, K. Asayama, Z. Hiroi, N. Kobayashi and M. Takano, Phys. Rev. B: Condens. Matter Mater. Phys., 1996, 53, R11942(R).

26 A. W. Garret, S. E. Nagler, T. Barnes and B. C. Sales, Phys. Rev. Lett., 1997, 81, 3968-3970.

27 C. Rovira, J. Veciana, E. Ribera, J. Tarrks, E. Canadell, R. Rousseau, E. Molins and M. Almeida, Angew. Chem., Int. Ed. Engl., 1997, 21, 2323-2326.

28 C. Rovira, Chem. - Eur. J., 2000, 6, 1723-1729.

29 C. Rovira, Molecular Compounds Showing a Spin Ladder Behaviour, in $\pi$-Electron Magnetism. Structure and Bonding, ed. J. Veciana and et al., Springer, Berlin, Heidelberg, 2001, vol. 100, pp. 163-188. 
30 C. Rovira, A. Pérez-Benítez, E. Molins, I. Mata, M. Almeida, H. Alves, J. Veciana, V. Gama, E. B. Lopes and R. T. Henriques, Synth. Met., 2003, 133-134, 523-526.

31 C. P. Landee and M. M. Turnbull, Eur. J. Inorg. Chem., 2013, 2266-2285.

32 E. Ribera, C. Rovira, J. Veciana, J. Tarrés, E. Canadell, R. Rousseau, E. Molins, M. Mas, J. P. Schoeffel, J. P. Pouget, J. Morgado, R. T. Henriques and M. Almeida, Chem. - Eur. J., 1999, 5, 2025-2039.

33 E. B. Lopes, R. T. Henriques, M. Almeida, E. Ribera, M. Mas-Torrent, J. Veciana, C. Rovira, P. Auban-Senzier and D. Jérome, Synth. Met., 2003, 133-134, 405-406.

34 R. Wesołowski, T. Haraldsen, L. Musfeldt, T. Barnes, M. Mas-Torrent, C. Rovira, T. Henriques and M. Almeida, Phys. Rev. B: Condens. Matter Mater. Phys., 2003, 68, 9-12.

35 D. Arćon, A. Lappas, S. Margadonna, K. Prassides, E. Ribera, J. Veciana, C. Rovira, R. T. Henriques and M. Almeida, Phys. Rev. B: Condens. Matter Mater. Phys., 1999, 60, 4191-4194.

36 E. Ribera, C. Rovira, J. Veciana, J. Tarrés, E. Canadell, R. Rousseau, E. Molins, M. Mas, J. P. Schoeffel, J.-P. Pouget, J. Morgado, V. Gama, R. T. Henriques and M. Almeida, Synth. Met., 1999, 102, 1743-1746.

37 X. Ribas, M. Mas-Torrent, A. Pérez-Benítez, J. C. Dias, H. Alves, E. B. Lopes, R. T. Henriques, E. Molins, I. C. Santos, K. Wurst, P. Foury-Leylekian, M. Almeida, J. Veciana and C. Rovira, $A d v$. Funct. Mater., 2005, 15, 1023-1035.

38 J. C. Dias, E. B. Lopes, I. C. Santos, M. T. Duarte, R. T. Henriques, M. Almeida, X. Ribas, C. Rovira, J. Veciana, P. Foury-Leylekian, J.-P. Pouget, P. Auban-Senzier and D. Jérome, J. Phys. IV, 2004, 114, 497-499.

39 R. A. L. Silva, I. C. Santos, J. Wright, J. T. Coutinho, L. C. J. Pereira, E. B. Lopes, S. Rabaça, J. Vidal-Gancedo, C. Rovira, M. Almeida and D. Belo, Inorg. Chem., 2015, 54, 7000-7006.

40 R. T. Clay and S. Mazumdar, J. Low Temp. Phys., 2006, 142, 365-370.

41 J. L. Musfeldt, S. Brown, S. Mazumdar, R. T. Clay, M. Mas-Torrent, C. Rovira, J. C. Dias, R. T. Henriques and M. Almeida, Solid State Sci., 2008, 10, 1740-1744.

42 E. B. Lopes, H. Alves, E. Ribera, M. Mas-Torrent, P. AubanSenzier, E. Canadell, R. T. Henriques, M. Almeida, E. Molins, J. Veciana, C. Rovira and D. Jérome, Eur. Phys. J. B, 2002, 29, 27-33.

43 R. A. L. Silva, A. I. S. Neves, E. B. Lopes, I. C. Santos, J. T. Coutinho, L. C. J. Pereira, C. Rovira, M. Almeida and D. Belo, Inorg. Chem., 2013, 52, 5300-5306.

44 V. Gama, R. T. Henriques, G. Bonfait, L. C. Pereira, J. C. Waerenborgh, I. C. Santos, M. T. Duarte, J. M. P. Cabral and M. Almeida, Inorg. Chem., 1992, 31, 2598-2604.

45 M. Almeida, V. Gama, I. C. Santos, D. Graf and J. S. Brooks, CrystEngComm, 2009, 11, 1103-1108.

46 J. V. Rodrigues, I. C. Santos, V. Gama, R. T. Henriques, J. C. Waerenborgh, M. T. Duarte and M. Almeida, J. Chem. Soc., Dalton Trans., 1994, 18, 2655-2660.

47 X. Ribas, A. Sironi, N. Masciocchi, E. B. Lopes, M. Almeida, J. Veciana and C. Rovira, Inorg. Chem., 2005, 44, 2358-2366.

48 T. Komatsu, N. Kojima and G. Saito, Solid State Commun., 1997, 103, 519-523.
49 T. Komatsu, N. Kojima and G. Saito, Synth. Met., 1999, 103, 1923-1924.

50 H. Nakajima, M. Katsuhara, H. Nii, M. Ashizawa, T. Kawamoto and T. Mori, Synth. Met., 2004, 145, 95-101.

51 M. Fourmigué, Acc. Chem. Res., 2004, 37, 179-186.

52 M. Fourmigué, B. Domercq, I. V. Jourdain, P. Molinié, F. Guyon and J. Amaudrut, Chem. - Eur. J., 1998, 4, 1714-1723.

53 B. Domercq, C. Coulon and M. Fourmigué, Inorg. Chem., 2001, 40, 371-378.

54 T. Otsuka, M. Yosimmaru, K. Awaga, H. Imai, T. Inabe, N. Wada and M. Ogata, J. Phys. Soc. Jpn., 2001, 70, 2711-2716.

55 H. Imai, T. Inabe, T. Otsuka, T. Okuno and K. Awaga, Phys. Rev. B: Condens. Matter Mater. Phys., 1996, 54, R6838-R6840.

56 H. Imai, T. Inabe, T. Otsuka, T. Okuno and K. Awaga, Synth. Met., 1997, 85, 1705-1706.

57 H. Imai, T. Otsuka, T. Naito, K. Awaga and T. Inabe, J. Am. Chem. Soc., 1999, 121, 8098-8103.

58 T. Otsuka, K. Awaga, H. Imai and T. Inabe, Synth. Met., 1999, 103, 2292-2293.

59 H. Zhang, B. Cai, H. B. Duan, G. J. Yuan, F. Xuan and X. M. Ren, Russ. J. Coord. Chem., 2013, 39, 147-155.

60 S. Nishihara, T. Akutagawa, T. Hasegawa and T. Nakamura, Chem. Commun., 2002, 408-409.

61 T. Akutagawa, D. Sato, Q. Ye, T. Endo, S. I. Noro, S. Takeda and T. Nakamura, Dalton Trans., 2010, 39, 8219-8227.

62 S. Nishihara, T. Akutagawa, T. Hasegawa, S. Fujiyama, T. Nakamura and T. Nakamura, J. Solid State Chem., 2002, 168, 661-667.

63 S. Nishihara, T. Akutagawa, T. Hasegawa, T. Nakamura, S. Fujiyama and T. Nakamura, Synth. Met., 2003, 137, 1279-1280.

64 K. Ichihashi, D. Konno, T. Date, T. Nishimura, K. Y. Maryunina, K. Inoue, T. Nakaya, K. Toyoda, Y. Tatewaki, T. Akutagawa, T. Nakamura and S. Nishihara, Chem. Mater., 2018, 30, 7130-7137.

65 K. Ichihashi, D. Konno, K. Y. Maryunina, K. Inoue, K. Toyoda, S. Kawaguchi, Y. Kubota, Y. Tatewaki, T. Akutagawa, T. Nakamura and S. Nishihara, Angew. Chem., Int. Ed., 2019, 58, 4169-4172.

66 S. Nishihara, T. Akutagawa, D. Sato, S. Takeda, S. I. Noro and T. Nakamura, Chem. - Asian J., 2007, 2, 1083-1090.

67 M. Fujisawa, A. Asakura, S. Okubo, H. Ohta, S. Nishihara, T. Akutagawa, T. Nakamura and Y. Hosokoshi, J. Phys.: Conf. Ser., 2009, 150, 3-7.

68 S. Nishihara, X. Zhang, K. Kunishio, K. Inoue, X. M. Ren, T. Akutagawa, J. I. Kishine, M. Fujisawa, A. Asakura, S. Okubo, H. Ohta and T. Nakamura, Dalton Trans., 2013, 42, 15263-15266.

69 M. Tamura, Y. Nakazawa, D. Shiomi, K. Nozawa, Y. Hosokoshi, M. Ishikawa, M. Takahashi and M. Kinoshita, Chem. Phys. Lett., 1991, 186, 401-404.

70 Y. Nakazawa, M. Tamura, N. Shirakawa, D. Shiomi, M. Takahashi, M. Kinoshita and M. Ishikawa, Phys. Rev. B: Condens. Matter Mater. Phys., 1992, 46, 8906-8914.

71 M. Tamura, Y. Hosokoshi, D. Shiomi, M. Kinoshita, Y. Nakasawa, M. Ishikawa, H. Sawa, T. Kitazawa, A. Eguchi, Y. Nishio and K. Kajita, J. Phys. Soc. Jpn., 2003, 72, 1735-1744. 
72 K. Lekin, J. W. L. Wong, S. M. Winter, A. Mailman, P. A. Dube and R. T. Oakley, Inorg. Chem., 2013, 52, 2188-2198.

73 K. Katoh, Y. Hosokoshi, K. Inoue and T. Goto, J. Phys. Soc. Jpn., 2000, 69, 1008-1011.

74 K. Katoh, Y. Hosokoshi, K. Inoue, M. I. Bartashevich, H. Nakano and T. Goto, J. Phys. Chem. Solids, 2002, 63, 1277-1280.

75 K. Mukai, Bull. Chem. Soc. Jpn., 1975, 8, 2381-2382.

76 T. Tonegawa, T. Nakao and M. Kaburagi, J. Phys. Soc. Jpn., 1996, 65, 3317-3330.

77 M. Hagiwara, Y. Narumi, K. Kindo, M. Kohno, H. Nakano, R. Sato and M. Takahashi, Phys. Rev. Lett., 1998, 80, 1312-1315.

78 T. Sakai, N. Okazaki, K. Okamoto, K. Kindo, Y. Narumi, Y. Hosokoshi, K. Kato, K. Inoue and T. Goto, Phys. Rev. B: Condens. Matter Mater. Phys., 2003, 329-333, 1203-1204.

79 T. Sakai, N. Okazaki, K. Okamoto, K. Kindo, Y. Narumi, Y. Hosokoshi, K. Kato, K. Inoue and T. Goto, Phys. Status Solidi Basic Res., 2003, 236, 429-432.

80 M. Kikuchi, K. Okamoto, K. Okunishi and T. Sakai, Prog. Theor. Phys. Suppl., 2005, 159, 251-255.

81 T. Sakai, K. Okamoto, K. Okunishi, K. Kindo, Y. Narumi, Y. Hosokoshi, K. Katoh, K. Inoue and T. Goto, Phys. Rev. B: Condens. Matter Mater. Phys., 2004, 346-347, 34-37.

82 H. Yamaguchi, T. Okubo, K. Iwase, T. Ono, Y. Kono, S. Kittaka, T. Sakakibara, A. Matsuo, K. Kindo and Y. Hosokoshi, Phys. Rev. B: Condens. Matter Mater. Phys., 2013, 88, 174410.
83 H. Yamaguchi, T. Okita, Y. Iwasaki, Y. Kono, N. Uemoto, Y. Hosokoshi, T. Kida, T. Kawakami, A. Matsuo and M. Hagiwara, Sci. Rep., 2020, 10, 9193.

84 H. Yamaguchi, K. Iwase, T. Ono, T. Shimokawa, H. Nakano, Y. Shimura, N. Kase, S. Kittaka, T. Sakakibara, T. Kawakami and Y. Hosokoshi, Phys. Rev. Lett., 2013, 110, 157205.

85 H. Yamaguchi, H. Miyagai, T. Shimokawa, K. Iwase, T. Ono, Y. Kono, N. Kase, K. Araki, S. Kittaka, T. Sakakibara, T. Kawakami, K. Okunishi and Y. Hosokoshi, J. Phys. Soc. Jpn., 2014, 83, 033707. 86 M. Roji and S. Miyashita, J. Phys. Soc. Jpn., 1996, 65, 883-886. 87 A. K. Kolezhuk and H.-J. Mikeska, Phys. Rev. B: Condens. Matter Mater. Phys., 1996, 53, R8848-R8850.

88 T. Vekua, G. I. Japaridze and H.-J. Mikeska, Phys. Rev. B: Condens. Matter Mater. Phys., 2003, 67, 064419.

89 T. Vekua, G. I. Japaridze and H.-J. Mikeska, Phys. Rev. B: Condens. Matter Mater. Phys., 2004, 70, 014425.

90 K. Hijii, A. Kitazawa and K. Nomura, Phys. Rev. B: Condens. Matter Mater. Phys., 2005, 72, 014449.

91 G. I. Japaridze, A. Langari and S. Mahdavifar, J. Phys.: Condens. Matter, 2007, 19, 076201.

92 H. Yamaguchi, H. Miyagai, M. Yoshida, M. Takigawa, K. Iwase, T. Ono, N. Kase, K. Araki, S. Kittaka, T. Sakakibara, T. Shimokawa, T. Okubo, K. Okunishi, A. Matsuo and Y. Hosokoshi, Phys. Rev. B: Condens. Matter Mater. Phys., 2014, 89, 220402.

93 H. Yamaguchi, H. Miyagai, Y. Kono, S. Kittaka, T. Sakakibara, K. Iwase, T. Ono, T. Shimokawa and Y. Hosokoshi, Phys. Rev. B: Condens. Matter Mater. Phys., 2015, 91, 125104. 